Research Article

\title{
The Spectrum of Mapping Ideals of Type Variable Exponent Function Space of Complex Variables with Some Applications
}

\author{
Awad A. Bakery $\mathbb{D}^{1,2}$ and Mustafa M. Mohammed $\mathbb{D D}^{1,3}$ \\ ${ }^{1}$ Department of Mathematics, University of Jeddah, College of Science and Arts at Khulis, Jeddah, Saudi Arabia \\ ${ }^{2}$ Department of Mathematics, Faculty of Science, Ain Shams University, Cairo, Egypt \\ ${ }^{3}$ Department of Statistics, Faculty of Science, Sudan University of Science \& Technology, Khartoum, Sudan
}

Correspondence should be addressed to Mustafa M. Mohammed; mustasta@gmail.com

Received 19 June 2021; Accepted 10 October 2021; Published 29 October 2021

Academic Editor: Sibel Yalçın

Copyright (c) 2021 Awad A. Bakery and Mustafa M. Mohammed. This is an open access article distributed under the Creative Commons Attribution License, which permits unrestricted use, distribution, and reproduction in any medium, provided the original work is properly cited.

\begin{abstract}
The topological and geometric behaviors of the variable exponent formal power series space, as well as the prequasi-ideal construction by $s$-numbers and this function space of complex variables, are investigated in this article. Upper bounds for $s$ -numbers of infinite series of the weighted $n$th power forward and backward shift operator on this function space are being investigated, with applications to some entire functions.
\end{abstract}

\section{Introduction}

Operator ideal theory has various applications in the geometry of Banach spaces, xed point theory, spectral theory, and other areas of mathematics, among other areas of knowledge. Throughout the article, we will adhere to the etymological conventions listed below. If any other sources are used, we will make a note of them.

1.1. Conventions 1.1. $\mathbb{N}=\{0,1,2, \cdots\}$.C: complex number space

$\mathbb{R}^{\mathbb{N}}$ : the space of all real sequences

$\ell_{\infty}$ : the space of bounded real sequences

$\ell^{r}$ : the space of $r$-absolutely summable real sequences

$c_{0}$ : the space of null real sequences

$e_{l}=(0,0, \cdots, 1,0,0, \cdots)$, as 1 lies at the $l^{\text {th }}$ coordinate, for all $l \in \mathbb{N}$

$\mathscr{F}$ : the space of each sequence with finite nonzero coordinates

card $(\mathfrak{G})$ : the number of elements of the set $\mathfrak{G}$

mi $\nearrow$ : the space of all monotonic increasing sequences of positive reals

$L$ : the ideal of all bounded linear operators between any arbitrary Banach spaces
$F$ : the ideal of finite rank operators between any arbitrary Banach spaces

$\Lambda$ : the ideal of approximable operators between any arbitrary Banach spaces

$L_{c}$ : the ideal of compact operators between any arbitrary Banach spaces

$L(\mathfrak{X}, \mathfrak{Y})$ : the space of all bounded linear operators from a Banach space $X$ into a Banach space $Y$

$L(\mathfrak{X})$ : the space of all bounded linear operators from a Banach space $X$ into itself

$F(\mathfrak{X}, \mathfrak{Y})$ : the space of finite rank operators from a Banach space $X$ into a Banach space $Y$

$F(\mathfrak{X})$ : the space of finite rank operators from a Banach space $X$ into itself

$\Lambda(\mathfrak{X}, \mathfrak{Y})$ : the space of approximable operators from a Banach space $X$ into a Banach space $Y$

$\Lambda(\mathfrak{X})$ : the space of approximable operators from a Banach space $X$ into itself

$L_{c}(\mathfrak{X}, \mathfrak{Y})$ : the space of compact operators from a Banach space $X$ into a Banach space $Y$

$L_{c}(\mathfrak{X})$ : the space of compact operators from a Banach space $X$ into itself

$\left(s_{a}(G)\right)_{a \in \mathbb{N}}$ : the sequence of $s$-numbers of the bounded linear operator $G$ 
$\left(\alpha_{a}(G)\right)_{a \in \mathbb{N}}$ : the sequence of approximation numbers of the bounded linear operator $G$

$\left(s_{a}(G)\right)_{a \in \mathbb{N}}$ : the sequence of Kolmogorov numbers of the bounded linear operator $G$

$S_{v}$ : the operator ideals formed by the sequence of $s$ -numbers in any sequence space $V$
$S_{V}^{\mathrm{app}}$ : the operator ideals formed by the sequence of approximation numbers in any sequence space $V$

$S_{V}^{K o l}$ : the operator ideals formed by the sequence of Kolmogorov numbers in any sequence space $V$

1.2. Notations 1.2 (see [1]). $S_{\mathscr{H}}:=\left\{S_{\mathscr{H}}(\mathfrak{X}, \mathfrak{Y}) ; \mathfrak{X}\right.$ and $\mathfrak{Y}$ are Banach Spaces $\}$, where

$$
S_{\mathscr{H}}(\mathfrak{X}, \mathfrak{Y}):=\left\{P \in L(\mathfrak{X}, \mathfrak{Y}): f_{s} \in \mathscr{H}, \text { where } f_{s}(z)=\sum_{n=0}^{\infty} s_{n}(P) z^{n} \text { converges for any } z \in \mathbb{C}\right\}
$$

$S_{\mathscr{H}}^{\text {app }}:=\left\{S_{\mathscr{H}}^{\text {app }}(\mathfrak{X}, \mathfrak{Y}) ; \mathfrak{X}\right.$ and $\mathfrak{Y}$ are Banach Spaces $\}$, where

$$
S_{\mathscr{H}}^{\text {app }}(\mathfrak{X}, \mathfrak{Y}):=\left\{P \in L(\mathfrak{X}, \mathfrak{Y}): f_{\text {app }} \in \mathscr{H}, \text { where } f_{\text {app }}(z)=\sum_{n=0}^{\infty} \alpha_{n}(P) z^{n} \text { converges for any } z \in \mathbb{C}\right\}
$$

$S_{\mathscr{H}}^{\mathrm{Kol}} ;\left\{S_{\mathscr{H}}^{\mathrm{Kol}}(\mathfrak{X}, \mathfrak{Y}) ; \mathfrak{X}\right.$ and $\mathfrak{Y}$ are Banach Spaces $\}$, where

$$
S_{\mathscr{H}}^{\mathrm{Kol}}(\mathfrak{X}, \mathfrak{Y}):=\left\{P \in L(\mathfrak{X}, \mathfrak{Y}): f_{\text {Kol }} \in \mathscr{H}, \text { where } f_{\text {Kol }}(z)=\sum_{n=0}^{\infty} d_{n}(P) z^{n} \text { converges for any } z \in \mathbb{C}\right\}
$$

$\left(S_{\mathscr{H}_{\rho}}\right)^{\lambda}:=\left\{\left(S_{\mathscr{H}_{\rho}}\right)^{\lambda}(\mathfrak{X}, \mathfrak{Y}) ; \mathfrak{X}\right.$ and $\mathfrak{Y}$ are Banach Spaces $\}$,

where

$$
\left(S_{\mathscr{H}_{\rho}}\right)^{\lambda}(\mathfrak{X}, \mathfrak{Y}):=\left\{T \in L(\mathfrak{X}, \mathfrak{Y}): f_{\lambda} \in \mathscr{H}_{\rho}, \text { where } f_{\lambda}(z)=\sum_{n=0}^{\infty} \lambda_{n}(T) z^{n} \text { converges for any } z \in \mathbb{C} \text { and }\left\|T-\lambda_{l}(T) I\right\|=0, \text { for every } l \in \mathbb{N}\right\}
$$

Several operator ideals in the class of Banach or Hilbert spaces are defined by sequences of real numbers. $L_{c}$, for example, is produced by $\left(d_{a}(G)\right)_{a \in \mathbb{N}}$ and $c_{0}$. Pietsch [2] looked into the quasi-ideals $S_{\ell^{t}}^{\text {app }}$, for $0<t<\infty$. He demonstrated how $\ell^{2}$ and $\ell^{1}$ yield the ideals of Hilbert Schmidt operators and nuclear operators between Hilbert spaces, respectively. In addition, he proved that $\bar{F}=S_{\ell^{t}}$, for $1 \leq t<$ $\infty$, and $S_{\ell^{t}}$ is a simple Banach space. Pietsch [3] explained that $S_{\ell^{t}}$, where $0<t<\infty$, is small. Makarov and Faried [4] showed that for any Banach spaces $\mathfrak{X}$ and $\mathfrak{Y}$ with $\operatorname{dim}(\mathfrak{X})$ $=\operatorname{dim}(\mathfrak{Y})=\infty$, then for every $r>t>0$, one has $S_{\mathfrak{\ell}^{t}}^{\text {app }}(\mathfrak{X}, \mathfrak{Y})$ )$\underset{\neq}{\subset S_{\ell^{r}}}$ app $\left._{(\mathfrak{X}}, \mathfrak{Y}\right) \underset{\neq}{\subset} L(\mathfrak{X}, \mathfrak{Y})$. The concept of prequasi-ideal was developed by Faried and Bakery [5], who elaborated on the concept of quasi-ideal. They investigated some geometric and topological properties of the spaces $S_{\operatorname{ces}(t)}$ and $S_{\ell_{M}}$. According to the spectral decomposition theorem [2], for $A \in L_{c}(H)$, where $H$ is a Hilbert space, one has $A(y)=\sum_{a=0}^{\infty}$ $\alpha_{a} \prec y, r_{a}>w_{a}$, where $\left\{r_{a}\right\}$ and $\left\{w_{a}\right\}$ are orthonormal families in $H$. Suppose $\left(t_{a}\right)_{a \in \mathbb{N}} \in \mathbb{R}^{\mathbb{N}}$ be decreasing and $D:\left(\eta_{a}\right)$ $\longrightarrow\left(t_{a} \eta_{a}\right)$ be the diagonal operator on $\ell^{p}$ with $p \geq 1$. Therefore, $s_{a}(D)=t_{a}$. Shields [6] investigated an indication to the weighted shift operators as formal power series in unilateral shifts and formal Laurent series in bilateral shifts. Hedayatian [7] offered the space of formal power series with power $r, \mathscr{H}^{r}((b a))$, where $((b a))$ is a sequence of positive numbers with $b_{0}=1$ and $r>0$. By the space $\mathscr{H}^{p}((b a))$, he meant that the set of all formal power series $\sum_{a=0}^{\infty} \widehat{f_{a}} z^{a}$ with $\sum_{a=0}^{\infty}$ 
$\left|b_{a} f_{a} \wedge\right|^{p}<\infty$. He studied cyclic vectors for the $H$ forward shift operator and supercyclic vectors for the backward shift operator on the space $\mathscr{H}^{p}\left(\left(b_{a}\right)\right)$.

However, Emamirad and Heshmati [8] explored the idea of functions evident on the Bargmann space by $f(z)=\sum_{a=0}^{\infty}$ $c_{a}\left(z^{a} / \sqrt{a !}\right)$ with $\|f\|=\sum_{a=0}^{\infty}\left|c_{a}\right|^{2}<\infty$, where $\left\{z^{a} / \sqrt{a !}: a \in\right.$ $\mathbb{N}\}$ is an orthonormal basis. Faried et al. [9] introduced the upper bounds for $s$-numbers of infinite series of the weighted $n$th power forward shift operator on $\mathscr{H}^{r}((b a))$, for $1 \leq r<\infty$, with some applications to some entire functions.

The paper is arranged as follows. In Section 3, we offer the definition of the space $\mathscr{H}_{p(\text {.) }}$ with definite function $\rho$. We introduce the sufficient conditions on $\mathscr{H}_{p(\text { (.) }}$ to generate premodular special space of formal power series. This gives that $\mathscr{H}_{p(.)}$ is a prequasinormed space. In Section 4, firstly, we give the sufficient conditions on $\mathscr{H}_{p(.)}$ such that the class $S_{\mathscr{H}}$ generates an operator ideal. Secondly, we explain enough settings (not necessary) on $\left(\mathscr{H}_{p(.)}\right)_{\rho}$, so that $\bar{F}=$ $S_{\left(\mathscr{H}_{p(.)}\right)_{\rho}}$. This shows the nonlinearity of $s$-type $\left(\mathscr{H}_{p(.)}\right)_{\rho}$ spaces which gives an answer of Rhoades [10] open problem. Thirdly, we investigate the conditions on $\left(\mathscr{H}_{p(.)}\right)_{\rho}$ such that the prequasi-ideal $S_{\left(\mathscr{H}_{p(.)}\right)}$ are Banach and closed. Fourthly, we examine the sufficient conditions on $\left(\mathscr{H}_{p(.)}\right)_{\rho}$ such that $S_{\left(\mathscr{\ell}_{p(.)}\right)_{\rho}}$ is strictly contained for different powers. We show the smallness of $S_{\left(\mathscr{H}_{p(.)}\right)_{p}}$. Fifthly, we investigate the simpleness of $S_{\left(\mathscr{C}_{p(.)}\right)_{\rho}}$. Sixthly, we present the enough setup on $\left(\mathscr{H}_{p(.)}\right)_{\rho}$ such that the class $L$ with its sequence of eigenvalues in $\left(\mathscr{H}_{p(.)}\right)_{\rho}$ equals $S_{\left(\mathscr{H}_{p(.)}\right)_{\rho}}$. In Section 5, we estimate the upper bounds for $s$-numbers of infinite series of the weighted $n$th power forward and backward shift operator on $\mathscr{H}_{p(.)}$ with approaches to some entire functions.

\section{Definitions and Preliminaries}

Definition 1 (see [11]). A function $s: L(\mathfrak{X}, \mathfrak{Y}) \longrightarrow[0, \infty)^{\mathbb{N}}$ is called an $s$-number, if the sequence $\left(s_{b}(B)\right)_{a=0}^{\infty}$, for all $B \in L$ $(\mathfrak{X}, \mathfrak{Y})$, shows the following settings:

(a) If $B \in L(\mathfrak{X}, \mathfrak{Y})$, then $\|B\|=s_{0}(B) \geq s_{1}(B) \geq s_{2}(B) \geq \cdots$ $\geq 0$

(b) $s_{b+a-1}\left(B_{1}+B_{2}\right) \leq s_{b}\left(B_{1}\right)+s_{a}\left(B_{2}\right)$, for every $B_{1}, B_{2} \in L$ $(\mathfrak{X}, \mathfrak{Y}), b, a \in \mathbb{N}$

(c) The inequality $s_{a}(A B D) \leq\|A\| s_{a}(B)\|D\|$ holds, if $D$ $\in L\left(\mathfrak{X}_{0}, \mathfrak{X}\right), B \in L(\mathfrak{X}, \mathfrak{Y})$ and $\left.A \in L(\mathfrak{Y}), \mathfrak{Y}_{0}\right)$, where $\mathfrak{X}_{0}$ and $\mathfrak{Y}_{0}$ are arbitrary Banach spaces

(d) Suppose $A \in L\left(\mathfrak{X}_{0}, \mathfrak{X}\right)$ and $\lambda \in \mathbb{R}$, then $s_{a}(\lambda A)=|\lambda|$ $s_{a}(A)$

(e) Let $\operatorname{rank}(A) \leq b$ then $s_{b}(A)=0$, whenever $A \in L($ $\left.\mathfrak{X}_{0}, \mathfrak{X}\right)$ (f) Assume $I_{\lambda}$ indicates the identity operator on the $\lambda$ -dimensional Hilbert space $\ell_{2}^{\lambda}$, then $s_{r \geq \lambda}\left(I_{\lambda}\right)=0$ or $s_{r<\lambda}\left(I_{\lambda}\right)=1$

Consider the following examples of $s$-numbers:

(i) The $b$ th approximation number, $\alpha_{b}(A)$, where

$$
\alpha_{b}(A)=\inf \{\|A-B\|: B \in L(X, Y) \text { and } \operatorname{rank}(B) \leq b\}
$$

(ii) The $b$ th Kolmogorov number, $d_{b}(A)$, where

$$
d_{b}(A)=\inf _{\operatorname{dim} Y \leq b} \sup _{\|u\| \leq 1} \inf _{v \in Y}\|A u-v\| .
$$

Remark 2 (see [11]). If $B \in L c(H)$, where $H$ be a Hilbert space, then all the $s$-numbers equal the eigenvalues of $|B|$, where $|B|=\sqrt{B * B}$.

Lemma 3 (see [2]). If $B \in L\left(\mathfrak{X}_{0}, \mathfrak{X}\right)$ and $B \notin \Lambda\left(\mathfrak{X}_{0}, \mathfrak{X}\right)$, then $D \in L(\mathfrak{X})$ and $M \in L(\mathfrak{Y})$ with $M B D_{e b}=e_{b}$, for each $b \in \mathbb{N}$.

Definition 4 (see [2]). A Banach space $\mathfrak{Y}$ ) is said to be simple if $L(\mathfrak{Y})$ has one and only one nontrivial closed ideal.

Theorem 5 (see [2]). If $D$ is a Banach space with $\operatorname{dim}(D)$ $=\infty$, then

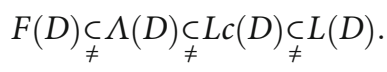

Definition 6. (see [2]). A class $U \subseteq L$ is said to be an operator ideal if every vector $U(X, Y)=U \cap L(X, Y)$ shows the following settings:

(i) $F \subseteq U$

(ii) $U(\mathfrak{X}, \mathfrak{Y})$ is linear space on $\mathbb{R}$

(iii) If $D \in L\left(\mathfrak{X}_{0}, \mathfrak{X}\right), B \in U(\mathfrak{X}, \mathfrak{Y}) \quad$ and $\quad A \in L\left(\mathfrak{Y}^{2}, \mathfrak{Y}_{0}\right)$ then, $A B D \in U\left(\mathfrak{X}_{0}, \mathfrak{Y}_{0}\right)$

Definition 7 (see [5]). A function $g: U \longrightarrow[0, \infty)$ is called a prequasinorm on the ideal $U$ if it shows the next settings:

(1) For each $A \in L(\mathfrak{X}, \mathfrak{Y}), g(A) \geq 0$ and $g(A)=0 \Leftrightarrow A$ $=0$

(2) One has $M \geq 1$ with $g(\beta A) \leq M|\beta| g(A)$, for all $\beta$ $\in \mathbb{R}$ and $A \in U(\mathfrak{X}, \mathfrak{Y})$

(3) One has $K \geq 1$ with $g\left(A_{1}+A_{2}\right) \leq K[g(A 1)+g(A 2)]$, for every $A_{1}, A_{2} \in U(\mathfrak{X}, \mathfrak{Y})$

(4) There exists $C \geq 1$ so that if $A \in L\left(\mathfrak{X}_{0}, \mathfrak{X}\right), B \in U(\mathfrak{X}$, $\mathfrak{Y})$ and $D \in L\left(\mathfrak{Y}, \mathfrak{Y}_{0}\right)$ then $g(D B A) \leq C\|D\| g(B)\|A\|$, where $\mathfrak{X}_{0}$ and $\mathfrak{Y}_{0}$ are normed spaces 
Theorem 8 (see [5]). Suppose $g$ is a quasinorm on the ideal $U$, then $g$ is a prequasinorm on the ideal $U$.

Theorem 9 (see [12]). Assume s-type $\mathscr{V} v:=\{f=(\operatorname{sr}(T)) \in$ $\mathbb{R}^{\mathbb{N}}: T \in L(\mathfrak{X}, \mathfrak{Y})$ and $\left.v(f)<\infty\right\}$. If $S v v$ is an operator ideal, then we have

(1) $\mathscr{F} \subset$ s-type $\mathscr{V} v$

(2) Assume $\left(s_{r}\left(T_{1}\right)\right)_{r=0}^{\infty} \in s$-type $\mathscr{V}_{v}$ and $\left(s_{r}\left(T_{2}\right)\right)_{r=0}^{\infty} \in s$ type $\mathscr{V}_{v}$, then $\left(s_{r}\left(T_{1}+T_{2}\right)\right)_{r=0}^{\infty} \in$ s-type $\mathscr{V}_{v}$

(3) Suppose $\lambda \in \mathbb{R}$ and $\left(s_{r}(T)\right)_{r=0}^{\infty} \in$ s-type $\mathscr{V}_{v}$, then $|\lambda|$ $\left(s_{r}(T)\right)_{r=0}^{\infty} \in$ s-type $\mathscr{V}_{v}$

(4) The sequence space $\mathscr{V}_{v}$ is solid. i.e., when $\left(s_{r}(G)\right)_{r=0}^{\infty}$ $\in$ s-type $\mathscr{V}_{v}$ and $s_{r}(T) \leq s_{r}(G)$, for every $r \in \mathbb{N}$ and $T$, $G \in L(X, Y)$, then $\left(s_{r}(T)\right)_{r=0}^{\infty} \in$ s-type $\mathscr{V}_{v}$

Lemma 10 (see [13]). If $\left\{\xi_{i}\right\}_{i \in \Psi}$ is a bounded family of $\mathbb{R}$. We have

$$
\sup _{\operatorname{card}(G)=a+1} \inf _{i \in G} \xi_{i}=\inf _{\operatorname{card}(G)=a} \sup _{i \notin G} \xi_{i} .
$$

Lemma 11 (see [14]). If $\left(r_{a}\right),\left(t_{a}\right) \in \mathbb{R}^{\mathbb{N}}$ and $\left(q_{a}\right) \in(0, \infty)^{\mathbb{N}}$, with $K=\max \left\{1,2^{\omega_{q-1}}\right\}$ and $\omega_{q}=\max \left\{1, \sup _{a} q_{a}\right\}$, then

$$
\left|r_{a}+t_{a}\right|^{q_{a}} \leq K\left(\left|r_{a}\right|^{q_{a}}+\left|t_{a}\right|^{q_{a}}\right)
$$

Definition 12 (see [1]). The linear space of formal power series

$$
\mathscr{H}=\left\{f: f(z)=\sum_{n=0}^{\infty} \widehat{f}_{n} z^{n} \text { converges for any } z \in \mathbb{C},\right\}
$$

is called a special space of formal power series (or in short (ssfps)), if it shows the following settings:

(1) $e^{(m)} \in \mathscr{H}$, for all $m \in \mathbb{N}$, where $e^{(m)}(z)=\sum_{n=0}^{\infty} e_{n}^{(\widehat{m})} z^{n}$ $=z^{m}$

(2) If $g \in \mathscr{H}$ and $\left|\widehat{f}_{n}\right| \leq\left|\widehat{g}_{n}\right|$, for all $n \in \mathbb{N}$, then $f \in \mathscr{H}$

(3) Suppose $f \in \mathscr{H}$, then $f_{[.]} \in \mathscr{H}$, where $f_{[.]}(z)=\sum_{b=0}^{\infty}$ $\widehat{f}_{[b / 2]} z^{b}$ and $[b / 2]$ marks the integral part of $b / 2$

Theorem 13 (see [1]). If $\mathscr{H}$ is a (ssfps), then $S_{\mathscr{H}}$ is an operator ideal.

By $\mathfrak{F}$, we explain the space of finite formal power series, i.e, for $f \in \mathfrak{F}$, one has $l \in \mathbb{N}$ with $f(z)=\sum_{n=0}^{l} \widehat{f}_{n} z^{n}$.

Definition 14 (see [1]). A subspace $\mathscr{H} \rho$ of the (ssfps) is called a premodular (ssfps), if there is a function $\rho: \mathscr{H} \longrightarrow[0, \infty)$ verifies the next conditions: (i) For $f \in \mathscr{H}$, we have $\rho(f) \geq 0$ and $f=\theta \Leftrightarrow \rho(f)=0$, where $\theta$ is the zero function of $\mathscr{H}$

(ii) Suppose $f \in \mathscr{H}$ and $\lambda \in \mathbb{R}$, then there is $l \geq 1$ with $\rho(\lambda f) \leq|\lambda| l \rho(f)$

(iii) Let $f, g \in \mathscr{H}$, then there is $K \geq 1$ such that $\rho(f+g$ )$\leq K(\rho(f)+\rho(g))$

(iv) Suppose $\left|\widehat{f}_{b}\right| \leq\left|\widehat{g}_{b}\right|$, for every $b \in \mathbb{N}$, then $\rho(f) \leq \rho$ (g)

(v) There is $K_{0} \geq 1$ so that $\rho(f) \leq \rho(f[].) \leq K_{0} \rho(f)$

(vi) $\overline{\mathfrak{F}}=\mathscr{H}_{\rho}$

(vii) one has $\xi>0$ with $\rho\left(\lambda e^{(0)}\right) \geq \xi|\lambda| \rho\left(e^{(0)}\right)$, where $\lambda$ $\in \mathbb{R}$

Note that the continuity of $\rho(f)$ at $\theta$ comes from condition (ii). Condition (1) in Definition 12 and condition (vi) in Definition 14 investigate that $\left(e^{(m)}\right)_{m} \in_{\mathbb{N}}$ is a Schauder basis of $\mathscr{H} \rho$.

The (ssfps) $\mathscr{H} \rho$ is called a prequasinormed (ssfps) if $\rho$ shows the conditions (i)-(iii) of Definition 14, and if the space $H$ is complete under $\rho$, then $\mathscr{H} \rho$ is called a prequasiBanach (ssfps).

Theorem 15 (see [1]). Every premodular (ssfps) $\mathscr{H} \rho$ is a prequasinormed (ssfps).

Definition 16 (see [1]). Assume $\mathscr{H} \rho$ is a prequasinormed (ssfps). An operator $V_{z}: \mathscr{H} \rho \longrightarrow \mathscr{H} \rho$ is called forward shift, if $V_{z} f=z f$, for all $f \in \mathscr{H}_{\rho}$, where $V_{z} f(z)=\sum_{n=0}^{\infty} \widehat{f}_{n}$ $z^{n+1}$ converges for every $z \in \mathbb{C}$ and $\rho\left(V_{z} f\right)<\infty$.

Definition 17 (see [1]). Suppose $\mathscr{H} \rho$ is a prequasinormed (ssfps). An operator $B_{z} \mathscr{H} \rho \longrightarrow \mathscr{H} \rho$ is called backward shift, if $B_{z} f(z)=(f(z)-f(0)) / z$, for all $f \in \mathscr{H} \rho$, where $B_{z} f$ $(z)=\sum_{n=0}^{\infty} \widehat{f}_{n+1} z^{n}$ converges for every $z \in \mathbb{C}$ and $\rho\left(B_{z} f\right)<$ $\infty$.

Definition 18 (see [9]). By using the power series of an entire function $g(z)=\sum_{m=0}^{\infty} a_{m} z^{m}$, the shift operator $V_{g(z)}$ is defined as

$$
V_{g(z)}(f(z))=\left(\sum_{m=0}^{\infty} a_{m} V_{z}^{m}\right)(f(z))
$$

Definition 19 (see [9]). By using the power series of an entire function $g(z)=\sum_{m=0}^{\infty} a_{m} z^{m}$, the shift operator $B_{g(z)}$ is defined as

$$
B_{g(z)}(f(z))=\left(\sum_{m=0}^{\infty} a_{m} B_{z}^{m}\right)(f(z))
$$




\section{Main Results}

3.1. The Space of Functions $\left(\mathscr{H}_{p(.)}\right)$. We define in this section the space $\left(\mathscr{H}_{p(.)}\right)_{\rho}$ under the function $\rho$ and give enough conditions on it to create pre-modular (ssfps) which implies that is a prequasi-Banach (ssfps).

If $p=\left(p_{v}\right)_{v \in \mathbb{N}}(0, \infty)^{\mathbb{N}}$, we define the new space of functions:

$$
\left(\mathscr{H}_{p(.)}\right)_{\rho}=\left\{f: f(z)=\sum_{v=0}^{\infty} \widehat{f}_{v} z^{v} \text { converges for any } z \in \mathbb{C} \text { and } \rho(\zeta f)<\infty \text {,for some } \zeta>0\right\} \text {, }
$$

where

$$
\begin{aligned}
\rho(f)=\sum_{v=0}^{\infty} \frac{1}{p_{v}}\left|\widehat{f}_{v}\right|^{p_{v}} . \quad(14) \\
\left(\mathscr{H}_{p(.))_{\rho}}=\left\{f: f(z)=\sum_{v=0}^{\infty} \widehat{f}_{v} z^{v} \text { converges for any } z \in \mathbb{C} \text { and } \rho(\zeta f)<\infty, \text { for some } \zeta>0\right\}\right. \\
=\left\{f: f(z)=\sum_{v=0}^{\infty} \widehat{f_{v}} z^{v} \text { converges for any } z \in \mathbb{C} \text { and } \sum_{v=0}^{\infty} \frac{1}{p_{v}}\left|\zeta \widehat{f}_{v}\right|^{p_{v}}<\infty \text {,for some } \zeta>0\right\} \\
=\left\{f: f(z)=\sum_{v=0}^{\infty} \widehat{f}_{v} z^{v} \text { converges for any } z \in \mathbb{C} \text { and } \inf _{v}|\zeta|^{p_{v}} \sum_{v=0}^{\infty} \frac{1}{p_{v}}\left|\zeta \widehat{f}_{v}\right|^{p_{v}}<\infty, \text { for some } \zeta>0\right\} \\
=\left\{f: f(z)=\sum_{v=0}^{\infty} \widehat{f}_{v} z^{v} \text { converges for any } z \in \mathbb{C} \text { and } \sum_{v=0}^{\infty} \frac{1}{p_{v}}\left|\widehat{f}_{v}\right|^{p_{v}}<\infty\right\} \\
=\left\{f: f(z)=\sum_{v=0}^{\infty} \widehat{f}_{v} z^{v} \text { converges for any } z \in \mathbb{C} \text { and } \rho(\zeta f)<\infty, \text { for any } \zeta>0\right\} .
\end{aligned}
$$

If $\left(p_{v}\right) \in \ell_{\infty}$, one has

Theorem 20. Consider $\left(p_{v}\right) \in m i, \cap \ell_{\infty}$ with $p_{0}>0$, one has $\left(\mathscr{H}_{p_{(.)}}\right)_{\rho}$ is a premodular Banach (ssfps).

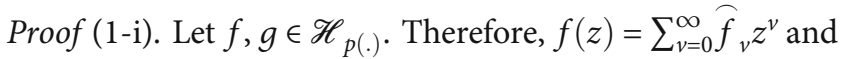
$g(z)=\sum_{v=0}^{\infty} \widehat{g}_{v} z^{v}$ converge for any $z \in \mathbb{C}$. Then, $(f+g)(z)$ $=\sum_{v=0}^{\infty}\left(\widehat{f}_{v}+\widehat{g}_{v}\right) z^{v}$ converges for any $z \in \mathbb{C}$. From $\left(p_{v}\right) \in \ell_{\infty}$, we

have-

$\sum_{v=0}^{\infty}\left(1 / p_{v}\right)\left|\widehat{f}_{v}+\widehat{g}_{v}\right|^{p_{v}} \leq K\left(\sum_{v=0}^{\infty}\left(1 / p_{v}\right)\left|\widehat{f}_{v}\right|^{p_{v}}+\sum_{v=0}^{\infty}\left(1 / p_{v}\right)\right.$ $\left.\left|\widehat{g}_{v}\right|^{p_{v}}\right)<\infty$,so $f+g \in \mathscr{H}_{p(.)}$.

(1-ii) Let $\lambda \in \mathbb{R}$ and $f \in \mathscr{H} p_{(.)}$. Therefore, $f(z)=\sum_{v=0}^{\infty} \widehat{f}_{v}$ $z^{v}$ converges for any $z \in \mathbb{C}$. Then, $(\lambda f)(z)=\sum_{v=0}^{\infty} \lambda \widehat{f}_{v} z^{v}$ con- verges for any $z \in \mathbb{C}$. From $\left(p_{v}\right) \in \ell_{\infty}$, we have $\sum_{v=0}^{\infty}\left(1 / p_{v}\right)\left|\lambda \widehat{f}_{v}\right|^{p_{v}} \leq \sup |\lambda|^{p_{v}} \sum_{v=0}^{\infty}\left(1 / p_{v}\right)\left|\widehat{f}_{v}\right|^{p_{v}}<\infty$.

So $\lambda f \in \mathscr{H}_{p(.)}$. Therefore, from conditions (1-i) and (1ii), the space $\mathscr{H}_{p(.)}$ is linear. To prove $e^{(m)} \in \mathscr{H}_{p_{(.)}}$,

for all $m \in \mathbb{N}$, where $e^{(m)}(z)=\sum_{v=0}^{\infty} e_{v}^{(\widehat{m})} z^{v}=z^{m}$ and $\sum_{v=0}^{\infty}($ $\left.1 / p_{v}\right)\left|e_{v}^{(\hat{m})}\right|^{p_{v}}=1 / p_{m}$

(2) Assume $\left|\widehat{f_{v}}\right| \leq\left|\widehat{g_{v}}\right|$, for all $v \in \mathbb{N}$ and $g \in \mathscr{H}_{p(.)}$. Then,converges for any $z \in \mathbb{C}$

One has

$$
\sum_{v=0}^{\infty} \frac{1}{p_{v}}\left|f \wedge_{v}\right|^{p_{v}} \leq \sum_{v=0}^{\infty} \frac{1}{p_{v}}\left|g \wedge_{v}\right|^{p_{v}}<\infty .
$$


So, $f(z)=\sum_{v=0}^{\infty} \widehat{f}_{v} z^{v}$ and $z \in \mathbb{C}$ and $\rho(f)<\infty$. Hence, $f$ $\in \mathscr{H}_{p(\cdot)}$

(3) Let $f \in \mathscr{H}_{p(\cdot)}$ and $\left(p_{v}\right) \in m i \nearrow \cap \ell_{\infty}$ with $p_{0}>0$. Then, $f(z)=\sum_{v=0}^{\infty} \widehat{f}_{v} z^{v}$ converges for any $z \in \mathbb{C}$

and $\rho(f)<\infty$. One has

$$
\begin{aligned}
\rho\left(f_{[\cdot]}\right)= & \sum_{v=0}^{\infty} \frac{1}{p_{v}}\left|f_{[v / 2]} \wedge\right|^{p_{v}}=\sum_{v=0}^{\infty} \frac{1}{p_{2 v}}\left|f_{v} \wedge\right|^{p_{2 v}} \\
& +\sum_{v=0}^{\infty} \frac{1}{p_{2 v+1}}\left|f_{v} \wedge\right|^{p_{2 v+1}} \leq 2 \sum_{v=0}^{\infty} \frac{1}{p_{v}}\left|f_{v} \wedge\right|^{p v}=2 \rho(f)
\end{aligned}
$$

Hence, $f_{[\cdot]}(z)=\sum_{v=0}^{\infty} \widehat{f_{[v / 2]}} z^{v}$ converges for any $z \in \mathbb{C}$ and $\rho\left(f_{[\cdot]}\right)<\infty$. Then $f_{[\cdot]} \in \mathscr{H}_{p(\cdot)}$.

(i) Obviously, if $f \in \mathscr{H}_{p(.)}$, one gets $\rho(f) \geq 0$ and $\rho(f)$ $=0 \Leftrightarrow f=\theta$

(ii) There is $l=\max \left\{1, \sup |\eta|^{p v-1}\right\} \geq 1$, for all $\eta \in \mathbb{R} \backslash\{$ $0\}$ and $l \geq 1$, for $\eta=0$ so that

$$
\rho(\eta f)=\sum_{v=0}^{\infty} \frac{1}{p_{v}}\left|\eta f_{v} \wedge\right|^{p_{v}} \leq \sup _{v}|\eta|^{p_{v}} \sum_{v=0}^{\infty} \frac{1}{p_{v}}\left|f_{v} \wedge\right|^{p_{v}} \leq l|\eta| \rho(f),
$$

for all $f \in \mathscr{H}_{p(.)}$

(iii) There is $K=\max \left\{1,2 \sup ^{\sup _{p_{v}}-1}\right\} \geq 1$ so that

$$
\rho(\eta f)=\sum_{v=0}^{\infty} \frac{1}{p_{v}}\left|\eta \widehat{f_{v}}\right| \leq \sup _{v}|\eta|^{p_{v}} \sum_{v=0}^{\infty} \frac{1}{p_{v}}\left|f_{v} \wedge\right|^{p_{v}} \leq l|\eta| \rho(f),
$$

for every $f, g \in \mathscr{H}_{p(.)}$

(iv) Obviously from the proof part (2).

(v) From the proof part (3), one has $K_{0}=2 \geq 1$

(vi) Clearly, $\overline{\mathfrak{F}}=\mathscr{H}_{p(.)}$

(vii) One has $\zeta$ with $0<\zeta \leq \eta^{p_{0}-1}$ with $\rho\left(\eta e^{(0)}\right) \geq \zeta|\eta| \rho($ $\left.e^{(0)}\right)$, for each $\eta \neq 0$ and $\zeta>0$, when $\eta=0$. Therefore, the space $\left(\mathscr{H}_{p(.)}\right)_{\rho}$ is a premodular (ssfps). To show that $\left(\mathscr{H}_{p(.)}\right)_{\rho}$ is a premodular Banach (ssfps), we suppose $f^{(i)}$ to be a Cauchy sequence in $\left(\mathscr{H}_{p(.)}\right)_{\rho}$, then for every $\varepsilon \in(0,1)$, there is $i_{0} \in N$ such that for all $i, j \geq i_{0}$, one gets

$$
\rho\left(f^{(i)}-f^{(j)}\right)=\sum_{v=0}^{\infty} \frac{1}{p_{v}}\left|f_{v}^{(i)} \wedge-f_{v}^{(j)} \wedge\right|^{p_{v}}<\varepsilon^{\Phi_{p}}
$$

For $i, j \geq i_{0}$ and $v \in \mathbb{N}$, we have

$$
\left|\widehat{f_{v}^{(i)}}-\widehat{f_{v}^{(j)}}\right|<\varepsilon
$$

So, $\left(\widehat{f_{v}^{(j)}}\right)$ is a Cauchy sequence in $\mathbb{R}$, for fixed $v \in \mathbb{N}$, hence $\lim _{j \rightarrow \infty} \widehat{f_{v}^{(j)}}=\widehat{f_{v}^{(0)}}$, for fixed $v \in \mathbb{N}$.

Therefore, $\rho\left(f^{(i)}-f^{(0)}\right)<\varepsilon^{\varrho} p$, for every $i \geq i_{0}$. Finally, to show that $f^{(0)} \in \mathscr{H}_{p(.)}$, we have

$\rho\left(f^{(0)}\right)=\rho\left(f^{(0)}-f^{(i)}+f^{(i)}\right) \leq K\left(\rho\left(f^{(i)}-f^{(0)}\right)+\rho\left(f^{(i)}\right)\right)<\infty$.

Hence, $f^{(0)} \in \mathscr{H}_{p(.)}$. Then, the space $\left(\mathscr{H}_{p(.)}\right)_{\rho}$ is a premodular Banach (ssfps).

In view of Theorems 15 and 20, we conclude the following theorem.

Theorem 21. If $\left(p_{v}\right) \in$ mi $\nearrow \cap \ell_{\infty}$ with $p_{0}>0$, then the space $\left(H_{p(.)}\right)_{\rho}$ is a prequasi-Banach (ssfps), where

$$
\rho(f)=\sum_{v=0}^{\infty}\left(1 / p_{v}\right)\left|f_{v} \wedge\right|^{p_{v}}, \text { for all } f \in \mathscr{H}_{p(\cdot)} .
$$

Theorem 22. Suppose $\left(p_{v}\right) \in$ mi $\nearrow \cap \ell_{\infty}$ with $p_{0}>0$, one has $\left(H_{p(.)}\right)_{\rho}$ is a prequasiclosed (ssfps), where

$$
\rho(f)=\sum_{v=0}^{\infty} \frac{1}{p_{v}}\left|f_{v} \wedge\right|^{p_{v}}, \text { for all } f \in \mathscr{H}_{p(\cdot)} .
$$

Proof. According to Theorem 21, the space $\left(H_{p(.)}\right)_{\rho}$ is a prequasinormed (ssfps). To explain that $\left(H_{p(.)}\right)_{\rho}$ is a prequasiclosed (ssfps), let $\left\{f^{(i)}\right\}_{i=0}^{\infty} \in\left(\mathscr{H}_{p(\cdot)}\right)_{\rho} \quad$ and $\lim _{i \rightarrow \infty} \rho\left(f^{(i)}-f^{(0)}\right)=0$, we have for all $\varepsilon \in(0,1)$, there is $i_{0} \in \mathbb{N}$ such that for all $i \geq i_{0}$, one gets

$$
\varepsilon>\rho\left(f^{(i)}-f^{(0)}\right)=\left[\sum_{a=0}^{\infty} \frac{1}{p_{a}}\left|f_{a}^{(i)} \wedge-f_{a}^{(0)} \wedge\right|^{p_{a}}\right]^{1 / \omega_{p}} .
$$

So, for $i \geq i_{0}$ and $a \in \mathbb{N}$, we have $\left|\widehat{f_{a}^{(i)}}-\widehat{f_{a}^{(0)}}\right|<\varepsilon$. Therefore, $\left(\widehat{f_{a}^{(i)}}\right)$ is a convergent sequence in $\mathbb{R}$, for fixed $a \in \mathbb{N}$. Then, $\lim _{i \rightarrow \infty} \widehat{f_{a}^{(i)}}=\widehat{f_{a}^{(0)}}$ for fixed $a \in \mathbb{N}$. Finally to prove 
that $f^{(0)} \in\left(\mathscr{H}_{p(.)}\right)$, we have

$$
\rho\left(f^{(0)}\right)=\rho\left(f^{(0)}-f^{(i)}+f^{(i)}\right) \leq \rho\left(f^{(i)}-f^{(0)}\right)+\rho\left(f^{(i)}\right)<\infty,
$$

this gives $f^{(0)} \in\left(\mathscr{H}_{p(.)}\right)_{\rho}$ which shows that $\left(\mathscr{H}_{p(.)}\right)_{\rho}$ is a prequasiclosed (ssfps).

\section{Properties of Operator Ideal}

Throughout this section, some geometric and topological properties of the prequasi-ideals formed by $s$-numbers and $\left(\mathscr{H}_{p(.)}\right)_{\rho}$ are presented.

4.1. Ideal of Finite Rank Operators. In this part, enough settings (not necessary) on $\left(\mathscr{H}_{p(.)}\right)_{\rho}$ so that $\bar{F}=S_{\left(\mathscr{H}_{p(.)}\right)_{\rho}}$ are given. This explains the nonlinearity of the $s$-type $\left(\mathscr{H}_{p(.)}\right)_{\rho}$ spaces (Rhoades open problem [10]).

In view of Theorems 13 and 20, we conclude the next theorem.

Theorem 23. Consider $\left(p_{v}\right) \in$ mi $\cap \cap \ell_{\infty}$ with $p_{0}>0$, then $S_{\mathscr{H}_{p(.)}}$ is an operator ideal.

Theorem 24. If $\left(p_{v}\right) \in$ mi $\cap \cap \ell_{\infty}$ with $p_{0}>0$, then $\bar{F}=S_{\left(\mathscr{L}_{p(.)}\right)_{\rho}}$ , where

$$
\rho(f)=\sum_{v=0}^{\infty} \frac{1}{p_{v}}\left|\widehat{f}_{v}\right|, \text { for every } f \in \mathscr{H}_{p(\cdot)} .
$$

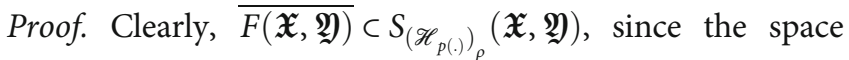
$S_{\left(\mathscr{C}_{P(.)}\right)_{\rho}}$ is an operator ideal. Therefore, we have to show that $S_{\left(\mathscr{H}_{p(\cdot)}\right)_{\rho}}(\mathfrak{X}, \mathfrak{Y}) \subseteq \overline{F(\mathfrak{X}, \mathfrak{Y})}$. By letting $T \in S_{\left(\mathscr{H}_{p(.)}\right)_{\rho}}(\mathfrak{X}, \mathfrak{Y})$, then, $f_{s} \in\left(H_{p(.)}\right)$, with $f_{s}(z)=\sum_{v=0}^{\infty} s_{v}(T) z^{v}$ converges for any $z$ $\in \mathbb{C}$. So, $\rho\left(f_{s}\right)<\infty$, fix $\varepsilon \in(0,1)$, we have $m \in \mathbb{N}-\{0\}$ with $\rho\left(f_{s}-\sum_{v=0}^{m-1} e^{(v)}\right)<\varepsilon / 4$. As $\left(s_{v}(T)\right)_{v \in \mathbb{N}}$ is decreasing, we have

$$
\sum_{v=m+1}^{2 m} \frac{1}{p_{v}}\left(s_{2 m}(T)\right)^{p_{v}} \leq \sum_{v=m+1}^{2 m} \frac{1}{p_{v}}\left(s_{v}(T)\right)^{p_{v}} \leq \sum_{v=m}^{\infty} \frac{1}{p_{v}}\left(s_{v}(T)\right)^{p_{v}}<\frac{\varepsilon}{4} .
$$

Therefore, we have $A \in F_{2 m}(\mathfrak{X}, \mathfrak{Y})$, rank $A \leq 2 m$ and

$$
\sum_{v=2 m+1}^{3 m} \frac{1}{p v}\|T-A\|^{p_{v}} \leq \sum_{v=m+1}^{2 m} \frac{1}{p_{v}}\|T-A\|^{p_{v}}<\frac{\varepsilon}{4} .
$$

As $\left(p_{v}\right) \in \ell_{\infty}$, then

$$
\sum_{v=0}^{m} \frac{1}{p_{v}}\|T-A\|^{p_{v}}<\frac{\varepsilon}{4}
$$

Since $T-A \in S_{\left(\mathscr{H}_{p(.)}\right)_{p}}(\mathfrak{X}, \mathfrak{Y})$, then $h_{s} \in\left(\mathscr{H}_{p(.)}\right)_{p}$, where $h_{s}(z):=\sum_{v=0}^{\infty} s_{v}(T-A) z^{v}$ converges for any $z \in \mathbb{C}$. Because $\left(p_{v}\right)$ is increasing and from the inequalities (27)-(29), we get

$$
\begin{aligned}
d(T, A) & =\rho\left(h_{s}\right)=\sum_{v=0}^{3 m-1} \frac{1}{p_{v}}\left(s_{v}(T-A)\right)^{p_{v}}+\sum_{v=3 m}^{\infty} \frac{1}{p_{v}}\left(s_{v}(T-A)\right)^{p_{v}} \\
& \leq \sum_{v=0}^{3 m} \frac{1}{p_{v}}\|T-A\|^{p_{v}}+\sum_{v=m}^{\infty} \frac{1}{p_{v+2 m}}\left(s_{v+2 m}(T-A)\right)^{p_{v+2 m}} \\
& \leq 3 \sum_{v=0}^{m} \frac{1}{p_{v}}\|T-A\|^{p_{v}}+\sum_{v=m}^{\infty} \frac{1}{p_{v}}\left(s_{v}(T)\right)^{p_{v}}<\varepsilon .
\end{aligned}
$$

Since $I_{2} \in S_{\left(\mathscr{H}_{(1 /(n+1))_{p}}\right)}(\mathfrak{X}, \mathfrak{Y})$ but the condition $\left(p_{v}\right) \in m i$ $\nearrow \cap \ell_{\infty}$ is not verified which explain a negative example of the converse statement. This finishes the proof.

We can reformulate Theorem 24 as follows: if $\left(p_{v}\right) \in m i$ $\neg \cap \ell_{\infty}$ with $p_{0}>0$, then every compact operators can be approximated by finite rank operators and the converse is not always true.

4.2. Banach and Closed Prequasi-Ideal. In this part, enough settings on $\left(\mathscr{H}_{p(.)}\right)_{\rho}$ so that the prequasioperator ideal $S_{\mathscr{H}_{\rho}}$ is Banach and closed are investigated.

Theorem 25. Assume $\left(p_{v}\right) \in$ mi $\cap \cap \ell_{\infty}$ with $p_{0}>0$, then the function $g(P)=\rho\left(f_{s}\right)$ is a prequasinorm on $S_{\left(\mathscr{H}_{p(.)}\right)}$, where $f_{s}(z)=\sum_{v=0}^{\infty} s_{v}(P) z^{v}$ converges for any $z \in \mathbb{C}$ and

$$
\rho\left(f_{s}\right)=\sum_{v=0}^{\infty} \frac{1}{p_{v}} s_{v}(P)^{p_{v}}, \text { for every } f_{s} \in \mathscr{H}_{p(\cdot)} .
$$

Proof. One has $g$ verifies the next setups:

(1) Let $P \in S_{\left(\mathscr{H}_{P(\cdot)}\right)_{\rho}}(\mathfrak{X}, \mathfrak{Y}), g(P)=\rho\left(f_{s}\right) \geq 0$ and $g(P)=\rho$ $\left(f_{s}\right)=0 \Leftrightarrow s_{v}(P)=0$, for all $v \in N \Leftrightarrow P=0$

(2) There is $l \geq 1$ with $g(\lambda P)=\rho\left(\lambda f_{s}\right) \leq l|\lambda| \rho\left(f_{s}\right)=l|\lambda|$ $g(P)$, for every $P \in S_{\left(\mathscr{H}_{P(.)}\right)_{\rho}}(\mathfrak{X}, \mathfrak{Y})$ and $\lambda \in \mathbb{R}$

(3) One has $K K_{0} \geq 1$, for $P_{1}, P_{2} \in S_{\left(\mathscr{H}_{p(.)}\right)_{\rho}}(\mathfrak{X}, \mathfrak{Y})$ ). Then, $f 1_{s}(z)=\sum_{v=0}^{\infty} s_{v}\left(P_{1}\right) z^{v} \quad$ and $\quad f 2_{s}(z)=\sum_{v=0}^{\infty} s_{v}\left(P_{2}\right) z^{v}$ converge for any $z \in \mathbb{C}$. Therefore, for $h_{s}(z)=: \sum_{v=0}^{\infty}$ $s_{v}\left(P_{1}+P_{2}\right) z^{v}$, one has

$$
\begin{aligned}
g\left(P_{1}+P_{2}\right)= & \rho\left(h_{s}\right) \leq \rho\left(\left(f 1_{s}\right)_{[\cdot]}+\left(f 2_{s}\right)_{[\cdot]}\right) \leq K\left(\rho\left(\left(f 1_{s}\right)_{[\cdot]}\right)\right. \\
& \left.+\rho\left(\left(f 2_{s}\right)_{[\cdot]}\right)\right) \leq K K_{0}\left(g\left(P_{1}\right)+g\left(P_{2}\right)\right)
\end{aligned}
$$


(4) We have $C \geq 1$, let $A \in L\left(\mathfrak{X}_{0}, \mathfrak{X}\right), B \in S_{\left(\mathscr{H}_{p(.)}\right)_{\rho}}(\mathfrak{X}, \mathfrak{Y})$ and $D \in L\left(\mathfrak{Y}, \mathfrak{Y}_{0}\right)$. Then, $f_{s}(z)=\sum_{v=0}^{\infty} s_{v}(B) z^{\nu}$ converges for all $z \in \mathbb{C}$. Then, for $h_{s}(z)=: \sum_{v=0}^{\infty} s_{v}(D B A)$ $z^{v}$, one has

$$
g(D B A)=\rho\left(h_{s}\right) \leq \rho\left(\|A\|\|D\| f_{s}\right) \leq C\|A\| g(B)\|D\|
$$

Theorem 26. Assume $\mathfrak{X}$ and $\mathfrak{Y}$ are Banach spaces, and ( $p_{y}$ )$\in$ mi $\nearrow \cap \ell_{\infty}$ with $p_{0}>0$, then $\left(S_{\left(\mathscr{H}_{p(.)}\right)}, g\right)$ is a prequasiBanach operator ideal, where $g(P)=\rho\left(f_{s}\right), f_{s}(z)=\sum_{v=0}^{\infty} s_{v}(P$ )$z^{v}$ converges for any $z \in \mathbb{C}$ and

$$
\rho\left(f_{s}\right)=\sum_{v=0}^{\infty} \frac{1}{p_{v}} s_{v}(P)^{p_{v}}, \text { for every } f_{s} \in \mathscr{H}_{p(\cdot)} .
$$

Proof. As $\left(p_{v}\right) \in m i \nearrow \cap \ell_{\infty}$ with $p_{0}>0$, one has the function

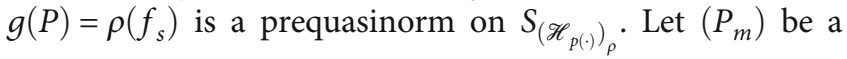
Cauchy sequence in $S_{\left(\mathscr{H}_{p(\cdot)}\right)_{\rho}}(\mathfrak{X}, \mathfrak{Y})$. Therefore, $f_{s}^{(m)} \in$ $\left(\mathscr{H}_{p(\cdot)}\right)_{\rho}$ and $f_{s}^{(m)}(z)=\sum_{v=0}^{\infty} s_{v}\left(P_{m}\right) z^{v}$ converges for any $z \in$ $\mathbb{C}$. Suppose $h_{s}(z)=: \sum_{v=0}^{\infty} s_{v}\left(P_{i}-P_{j}\right) z^{v}$, then from parts (iv) and (vii) of Definition 14 and since $\left.L(\mathfrak{X}, \mathfrak{Y}) \supseteq S_{(\mathscr{H}}(\cdot)\right)_{\rho}(\mathfrak{X}, \mathfrak{V}$ ), we have

$$
\begin{aligned}
g\left(P_{i}-P_{j}\right) & =\rho\left(h_{s}\right) \geq \rho\left(s_{0}\left(P_{i}-P_{j}\right) e^{(0)}\right)=\rho\left(\left\|P_{i}-P_{j}\right\| e^{(0)}\right) \\
& \geq \xi\left\|P_{i}-P_{j}\right\| \rho\left(e^{(0)}\right)
\end{aligned}
$$

then $\left(P_{m}\right)_{m \in \mathbb{N}}$ is a Cauchy sequence in $L(\mathfrak{X}, \mathfrak{Y})$. Since the space $L(\mathfrak{X}, \mathfrak{Y})$ is a Banach space, there is $P \in L(\mathfrak{X}, \mathfrak{Y})$ with $\lim _{m \longrightarrow \infty}\left\|P_{m}-P\right\|=0$ and as $f_{s}^{(m)} \in\left(\mathscr{H}_{p(.)}\right)$, for every $m \in \mathbb{N}$. Hence, by using Theorem 25 and the continuity of $\rho$ at $\theta$, we have

$$
\begin{aligned}
g(P) & =g\left(P-P_{m}+P_{m}\right) \leq K K_{0}\left(g\left(P_{m}-P\right)+g\left(P_{m}\right)\right) \\
& =K K_{0} \rho\left(\left\|P_{m}-P\right\| \sum_{m=-}^{\infty} e^{(m)}\right)+K K_{0} \rho\left(f_{s}^{(m)}\right)<\varepsilon,
\end{aligned}
$$

so $f_{s} \in\left(\mathscr{H}_{p(.)}\right)_{\rho}$, which implies $P \in S_{\left(\mathscr{\ell}_{p(.)}\right)_{\rho}}(\mathfrak{X}, \mathfrak{Y})$.

Theorem 27. Suppose $\mathfrak{X}$ and $\mathfrak{Y}$ are Banach spaces, and ( $p_{v}$ )$\in$ mi $\cap \cap \ell_{\infty}$ with $p_{0}>0$, then $\left(S_{\left(\mathscr{H}_{p(.)}\right)}, g\right)$ is a prequasiclosed operator ideal, where $g(P)=\rho\left(f_{s}\right), f_{s}(z)=\sum_{v=0}^{\infty} s_{v}(P) z^{v}$ con- verges for any $z \in \mathbb{C}$ and

$$
\rho\left(f_{s}\right)=\sum_{v=0}^{\infty} \frac{1}{p_{v}} s_{v}(P)^{p_{v}}, \text { for every } f_{s} \in \mathscr{H}_{p(\cdot)} .
$$

Proof. As $\left(p_{v}\right) \in m i \nearrow \cap \ell_{\infty}$ with $p_{0}>0$, so the function $g(P)$ $=\rho\left(f_{s}\right)$ is a prequasinorm on $S_{\left(\mathscr{H}_{p(.)}\right)_{\rho}}$. Let $P_{m} \in S_{\left(\mathscr{H}_{p(.)}\right)_{\rho}}(\mathfrak{X}$, $\mathfrak{Y})$, with $m \in \mathbb{N}$ and $\lim _{m \longrightarrow \infty} g\left(P_{m}-P\right)=0$. Then, $f_{s}^{(m)} \in$ $\left(\mathscr{H}_{p(.)}\right)_{\rho}$ and $f_{s}^{(m)}(z)=\sum_{v=0}^{\infty} s_{v}\left(P_{m}\right) z^{v}$ converges for any $z \epsilon$ $\mathbb{C}$. Suppose $h_{s}(z)=: \sum_{v=0}^{\infty} s_{v}\left(P_{i}-P_{j}\right) z^{v}$, then from parts (iv) and (vii) of Definition 14 and since $L(\mathfrak{X}, \mathfrak{V}) \supseteq S_{\left(\mathscr{H}_{p(.)}\right)_{\rho}}(\mathfrak{X}, \mathfrak{Y})$ ), one obtains

$$
\begin{aligned}
g\left(P-P_{j}\right) & =\rho\left(h_{s}\right) \geq \rho\left(s_{0}\left(P-P_{j}\right) e^{(0)}\right)=\rho\left(\left\|P-P_{j}\right\| e^{(0)}\right) \\
& \geq \xi\left\|P-P_{j}\right\| \rho\left(e^{(0)}\right)
\end{aligned}
$$

then $\left(P_{m}\right)_{m \in \mathbb{N}}$ is a convergent sequence in $L(\mathfrak{X}, \mathfrak{Y})$. Since the space $L(\mathfrak{X}, \mathfrak{Y})$ is a Banach space, then there is $P \in L(\mathfrak{X}, \mathfrak{Y})$ with $\lim _{m \longrightarrow \infty}\left\|P_{m}-P\right\|=0$ and as $f_{s}^{(m)} \in\left(\mathscr{H}_{p(.)}\right)$, for every $m \in \mathbb{N}$, by using Theorem 25 and the continuity of $\rho$ at $\theta$, one has

$$
\begin{aligned}
g(P) & =g\left(P-P_{m}+P_{m}\right) \leq K K_{0}\left(g\left(P_{m}-P\right)+g\left(P_{m}\right)\right) \\
& =K K_{0} \rho\left(\left\|P_{m}-P\right\| \sum_{m=0}^{\infty} e^{(m)}\right)+K K_{0} \rho\left(f_{s}^{(m)}\right)<\varepsilon,
\end{aligned}
$$

hence, $f_{s} \in\left(\mathscr{H}_{p(.)}\right)_{p}$, which gives $P \in S_{\left(\mathscr{H}_{p(.)}\right)_{p}}(\mathfrak{X}, \mathfrak{Y})$.

According to Theorem 9, we introduce the following properties of the $s$-type $\left(\mathscr{H}_{p(.)}\right)$.

Theorem 28. For s-type $\left(H_{p(.)}\right)_{\rho}:=\left\{\left(s_{v}(T)\right) \in \mathbb{R}^{\mathbb{N}}: T \in\right.$ $\left.S_{\left(\mathscr{H}_{p(.)}\right)_{\rho}}(\mathfrak{X}, \mathfrak{V})\right\}$. The next settings are verified.

(1) We have s-type $\left(\mathscr{H}_{p(.)}\right)_{\rho} \supset \mathscr{F}$

(2) Suppose $\left(s_{r}\left(T_{1}\right)\right)_{r=0}^{\infty} \in$ s-type $\left(\mathscr{H}_{p(\cdot)}\right)_{\rho}$ and $\left(s_{r}\left(T_{2}\right)\right)_{r=0}^{\infty}$ $\in$ s-type $\left(\mathscr{H}_{p(\cdot)}\right)_{\rho}$, then $\left(s_{r}\left(T_{1}+T_{2}\right)\right)_{r=0}^{\infty} \in$ s-type $\left(\mathscr{H}_{p(.)}\right)_{\rho}$

(3) One has $\lambda \in \mathbb{R}$ and $\left(s_{r}(T)\right)_{r=0}^{\infty} \in s$-type $\left(\mathscr{H}_{p(\cdot)}\right)_{\rho}$, then $|\lambda|\left(s_{r}(T)\right)_{r=0}^{\infty} \in s-\operatorname{type}\left(\mathscr{H}_{p(.)}\right)_{\rho}$

(4) The s-type $\left(\mathscr{H}_{p(.)}\right)$ is solid 
4.3. Small Prequasi-Banach Ideal. We introduce here some inclusion relations concerning the space $S_{\left(\mathscr{H}_{p(\bullet)}\right)}$ for different $\left(p_{v}\right)$.

Theorem 29. Let $\mathfrak{X}$ and $\mathfrak{Y}$ be Banach spaces with $\operatorname{dim}(\mathfrak{X})$ $=\operatorname{dim}(\mathfrak{Y})=\infty$, and $\left(p_{v}\right),\left(q_{v}\right) \in$ mi $\cap \cap \ell_{\infty}$ with $p_{0}>0$ and $p_{v}<q_{v}$, for all $v \in \mathbb{N}$, we have

$$
S_{\left(\mathscr{H}_{p(\cdot)}\right)_{\rho}}(\mathfrak{X}, \mathfrak{Y}) \underset{\neq}{\subset} S_{\left(\mathscr{H}_{p(\cdot)}\right)_{\rho}}(\mathfrak{X}, \mathfrak{Y}) \underset{\neq}{\subset} L(\mathfrak{X}, \mathfrak{Y}) .
$$

Proof. Assume $T \in S\left(\mathscr{H}_{p(.)}\right)_{\rho}(\mathfrak{X}, \mathfrak{Y})$. Therefore, $f_{s} \in\left(\mathscr{H}_{p(\cdot)}\right)_{\rho}$ and $f_{s}(z)=\sum_{v=0}^{\infty} s_{v}(T) z^{v}$ converges for any $z \in \mathbb{C}$. Then,

$$
\sum_{v=0}^{\infty} \frac{1}{q_{v}}\left(s_{v}(T)\right)^{q_{v}}<\sum_{v=0}^{\infty} \frac{1}{p_{v}}\left(s_{v}(T)\right)^{q_{v}}<\infty,
$$

hence, $T \in S\left(\mathscr{H}_{q(.)}\right)_{\rho}(\mathfrak{X}, \mathfrak{Y})$. Next, by taking $T$ with $s_{v}(T)=$ $\left(p_{v} /(v+1)\right)^{1 / p_{v}}$, one has $T \notin S\left(\mathscr{H}_{p(.)}\right)_{\rho}(\mathfrak{X}, \mathfrak{Y})$ and $T \in S$ $\left(\mathscr{H}_{q(.)}\right)_{\rho}(\mathfrak{X}, \mathfrak{Y})$. Clearly, $S_{\left(H_{q(.)}\right)_{\rho}}(\mathfrak{X}, \mathfrak{Y}) \subset L(\mathfrak{X}, \mathfrak{Y})$. Again, by choosing $s_{v}(T)=\left(q_{v} /(v+1)\right)^{1 / q_{v}}$, one has $T \notin S\left(\mathscr{H}_{q(.)}\right)_{\rho}(\mathfrak{X}$, $\mathfrak{Y})$ and $T \in L(\mathfrak{X}, \mathfrak{Y})$. This finishes the proof.

In this part, we examine the sufficient setting for which $S_{\left(\mathscr{H}_{p(\cdot)}\right)_{\rho}}^{a p p}$ is small.

Theorem 30. Let $\mathfrak{X}$ and $\mathfrak{Y}$ be Banach spaces with $\operatorname{dim}(\mathfrak{X})$ $=\operatorname{dim}(\mathfrak{Y})=\infty$. Assume $\left(p_{v}\right) \in$ mi $\backslash \cap \ell_{\infty}$ with $p_{0}>0$, then $S_{\left(\mathscr{H}_{p(\cdot)}^{a p p}\right)}$ is small.

Proof. Obviously, the space $\left(S_{\left(\mathscr{H}_{p(\cdot)}\right)_{\rho}}^{\text {app }}, g\right)$ generates a prequasiBanach operator ideal, with $g(T)=\sum_{v=0}^{\infty}\left(1 / p_{v}\right)\left(\alpha_{v}(T)\right)^{p_{v}}$. Let $S_{\left(\mathscr{H}_{p(\cdot)}\right)_{\rho}}^{\text {app }}(\mathfrak{X}, \mathfrak{Y})=L(\mathfrak{X}, \mathfrak{Y})$. Hence, there is $C>0$ with $g(T)$ $\leq C\|T\|$, for all $T \in L(\mathfrak{X}, \mathfrak{Y})$. According to Dvoretzky's theorem [15] with $r \in \mathbb{N}$, there are quotient spaces $\mathfrak{X} / \lambda_{r}$ and subspaces $\eta_{r}$ of $\mathfrak{Y}$ that operated onto $\ell_{2}^{r}$ by isomorphisms $D_{r}$ and $B_{r}$ with $\left\|D_{r}\right\|\left\|D_{r}^{-1}\right\| \leq 2$ and $\left\|B_{r}\right\|\left\|B_{r}^{-1}\right\| \leq 2$. Suppose $I_{r}$ be the identity operator on $\ell_{2}^{r}, \zeta_{r}$ be the quotient operator from $\mathfrak{X}$ onto $\mathfrak{X} / \lambda_{r}$ and $J_{r}$ be the natural embedding operator from $\eta_{r}$ into $\mathfrak{Y}$. Let $h_{a}$ be the Bernstein numbers [16], we have

$$
\begin{aligned}
1 & =h_{a}\left(I_{r}\right)=h_{a}\left(B_{r} B_{r}^{-1} I_{r} D_{r} D_{r}^{-1}\right) \leq\left\|B_{r}\right\| h_{a}\left(B_{r}^{-1} I_{r} D_{r}\right)\left\|D_{r}^{-1}\right\| \\
& =\left\|B_{r}\right\| h_{a}\left(J_{r} B_{r}^{-1} I_{r} D_{r}\right)\left\|D_{r}^{-1}\right\| \leq\left\|B_{r}\right\| d_{a}\left(J_{r} B_{r}^{-1} I_{r} D_{r}\right)\left\|D_{r}^{-1}\right\| \\
& =\left\|B_{r}\right\| d_{a}\left(J_{r} B_{r}^{-1} I_{r} D_{r} \zeta_{r}\right)\left\|D_{r}^{-1}\right\| \leq\left\|B_{r}\right\| \alpha_{a}\left(J_{r} B_{r}^{-1} I_{r} D_{r} \zeta_{r}\right)\left\|D_{r}^{-1}\right\|,
\end{aligned}
$$

for $0 \leq j \leq r$. Then for $l \geq 1$, one has

$$
\begin{aligned}
\frac{1}{p_{j}} & \leq\left(\left\|B_{r}\right\|\left\|D_{r}^{-1}\right\|\right)^{p_{j}} \frac{1}{p_{j}}\left(\alpha_{j}\left(J_{r} B_{r}^{-1} I_{r} D_{r} \zeta_{r}\right)\right)^{p_{j}} \Rightarrow \frac{1}{p_{j}} \\
& \leq l\left\|B_{r}\right\| \frac{1}{p_{j}}\left(\alpha_{j}\left(J_{r} B_{r}^{-1} I_{r} D_{r} \zeta_{r}\right)\right)^{p_{j}}\left\|D_{r}^{-1}\right\| \Rightarrow \sum_{j=0}^{r} \frac{1}{p_{j}} \\
& \leq l\left\|B_{r}\right\|\left\|D_{r}^{-1}\right\| \sum_{j=0}^{r} \frac{1}{p_{j}}\left(\alpha_{j}\left(J_{r} B_{r}^{-1} I_{r} D_{r} \zeta_{r}\right)\right)^{p_{j}} \Rightarrow \sum_{j=0}^{r} \frac{1}{p_{j}} \\
& \leq l C\left\|B_{r}\right\|\left\|D_{r}^{-1}\right\| g\left(J_{r} B_{r}^{-1} I_{r} D_{r} \zeta_{r}\right) \Rightarrow \sum_{j=0}^{r} \frac{1}{p_{j}} \\
& \leq l C\left\|B_{r}\right\|\left\|D_{r}^{-1}\right\|\left\|J_{r} B_{r}^{-1} I_{r} D_{r} \zeta_{r}\right\| \Rightarrow \sum_{j=0}^{r} \frac{1}{p_{j}} \\
& \leq l C\left\|B_{r}\right\|\left\|D_{r}^{-1}\right\|\left\|J_{r} B_{r}^{-1}\right\|\left\|I_{r}\right\|\left\|D_{r} \zeta_{r}\right\| \\
& =l C\left\|B_{r}\right\|\left\|D_{r}^{-1}\right\|\left\|B_{r}^{-1}\right\|\left\|I_{r}\right\|\left\|D_{r}\right\| \Rightarrow \sum_{j=0}^{r} \frac{1}{p_{j}} \leq 4 l C .
\end{aligned}
$$

As $r \longrightarrow \infty$, we get $\sum_{j=0}^{\infty} 1 / p_{j}<\infty$. Since $\sum_{j=0}^{\infty} a / p_{j} \geq 1 /$ $\sup p_{j} \sum_{j=0}^{\infty} 1=\infty$. Hence, the space $S_{\left(\mathscr{H}_{p(\cdot)}\right)_{p}}^{\text {app }}$ is small.

By the same manner, we can easily conclude the next theorem.

Theorem 31. Assume $\mathfrak{X}$ and $\mathfrak{Y}$ be Banach spaces with dim $(\mathfrak{X})=\operatorname{dim}(\mathfrak{Y})=\infty$. Suppose $\left(p_{v}\right) \in$ mi $\cap \cap \ell_{\infty}$ with $p_{0}>0$, then $S_{\left(\mathscr{H}_{p(\cdot)}\right)_{\rho}}^{\mathrm{Kol}}$ is small.

4.4. Simple Prequasi-Ideal. In this part, we offer enough settings on $\left(\mathscr{H}_{p(\cdot)}\right)_{\rho}$ so that the space $S_{\left(\mathscr{H}_{p(\cdot)}\right)_{\rho}}$ is simple.

Theorem 32. Let $\left(p_{v}\right),\left(q_{v}\right) \in$ mi $\cap \ell_{\infty}$ with $1 \leq p_{v}<q_{v}$, for every $v \in \mathbb{N}$, then

$$
L\left(S_{\left(\mathscr{H}_{q(\cdot)}\right)_{\rho}}, S_{\left(\mathscr{H}_{p(\cdot)}\right)_{\rho}}\right)=\Lambda\left(S_{\left(\mathscr{H}_{q(\cdot)}\right)_{\rho}}, S_{\left(\mathscr{H}_{p(\cdot)}\right)_{\rho}}\right)
$$

Proof. Consider $T \in L\left(S_{\left(\mathscr{H}_{q(\cdot)}\right)_{\rho}}, S_{\left(\mathscr{H}_{p(\cdot)}\right)_{\rho}}\right)$ and $T \notin \Lambda\left(S_{\left(\mathscr{H}_{q(\cdot)}\right)_{\rho}}\right.$,

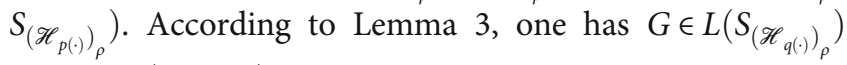
and $B \in L\left(S_{\left(\mathscr{H}_{p(\cdot)}\right)_{\rho}}\right)$ with BTGI ${ }_{m}=I_{m}$. For every $m \in \mathbb{N}$, one obtains

$$
\begin{aligned}
& \left\|I_{m}\right\|_{S_{\left(\mathscr{H}_{p(\cdot)}\right)_{\rho}}}=\left(\sum_{v=0}^{\infty} \frac{1}{p_{v}}\left(s_{v}\left(I_{m}\right)\right)^{p_{v}}\right)^{1 / \text { sup } p_{v}}=\left(\sum_{v=0}^{m-1} \frac{1}{p_{v}}\right)^{1 / \text { sup } p_{v}} \\
& \leq\|B T G\|\left\|I_{m}\right\|_{S_{\left(\mathscr{H}_{q(\cdot)}\right)_{\rho}}} \leq\left(\sum_{v=0}^{\infty} \frac{1}{q_{v}}\left(s_{v}\left(I_{m}\right)\right)^{q_{v}}\right)^{1 / \text { sup } q_{v}} \\
& =\left(\sum_{v=0}^{m-1} \frac{1}{q_{v}}\right)^{1 / \text { sup } q_{v}} .
\end{aligned}
$$

This defies Theorem 29. 
Corollary 33. Let $\left(p_{v}\right),\left(q_{v}\right) \in$ mi $\cap \cap \ell_{\infty}$ with $1 \leq p_{v}<q_{v}$, for each $v \in \mathbb{N}$, then

$$
L\left(S_{\left(\mathscr{H}_{q(\cdot)}\right)_{p}}, S_{\left(\mathscr{H}_{P(\cdot)}\right)_{p}}\right)=L_{C}\left(S_{\left(\mathscr{H}_{q(\cdot)}\right)_{p}}, S_{\left(\mathscr{H}_{P(\cdot)}\right)_{p}}\right) .
$$

Proof. Clearly, as $\Lambda \subseteq L_{c}$.

Theorem 34. Assume $\left(p_{v}\right) \in$ mi $\cap \ell_{\infty}$ with $p_{0} \geq 1$, then $S_{\left(\mathscr{\ell}_{p(.)}\right)_{\rho}}$ is simple.

Proof. Suppose $T \in L_{C}\left(S_{\left(\mathscr{H}_{P(\cdot)}\right)_{P}}\right)$ and $T \notin \Lambda\left(S_{\left(\mathscr{H}_{p(\cdot)}\right)_{P}}\right)$. In view of Lemma 3, we have $G, B \in L\left(S_{\left(\mathscr{\ell}_{P(\cdot)}\right)_{\rho}}\right)$ so as to $B T G I_{k}=I_{k}$.

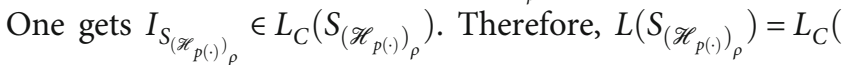
$S_{\left.\left(\mathscr{C}_{P(.)}\right)_{\rho}\right)}$. This implies one and only one nontrivial closed ideal $\Lambda\left(S_{\left(\mathscr{H}_{P(\cdot)}\right)_{\rho}}\right)$ in $L\left(S_{\left(\mathscr{H}_{p(\cdot)}\right)_{\rho}}\right)$.

4.5. Spectrum of Prequasi-Ideal. In this part, we introduce enough settings on $\left(\mathscr{H}_{p(\cdot)}\right)_{\rho}$ so that the class $L$ with sequence of eigenvalues in $\left(\mathscr{H}_{p(\cdot)}\right)_{\rho}$ equals $S_{\left(\mathscr{H}_{p(\cdot)}\right)_{\rho}}$.

Theorem 35. If $\mathfrak{X}$ and $\mathfrak{Y}$ are Banach spaces with $\operatorname{dim}(\mathfrak{X})$ $=\operatorname{dim}(\mathfrak{Y})=\infty$. Suppose $\left(p_{v}\right) \in$ mi $>\cap \ell_{\infty}$ with $p_{0}>0$, we have

$$
\left(S_{\left(\mathscr{H}_{P()}\right)_{\rho}}\right)^{\lambda}(\mathfrak{X}, \mathfrak{Y})=S_{\left(\mathscr{H}_{P()}\right)_{\rho}}(\mathfrak{X}, \mathfrak{Y}) .
$$

Proof. Let $T \in\left(S_{\left(\mathscr{H}_{p(\cdot)}\right)_{\rho}}\right)^{\lambda}(\mathfrak{X}, \mathfrak{Y})$, then $f_{\lambda} \in\left(\left(\mathscr{H}_{p(\cdot)}\right)_{\rho}\right)$, where $f_{\lambda}(z)=\sum_{v=0}^{\infty} \lambda_{v}(T) z^{\nu}$ converges for all $z \in \mathbb{C}$ with $\rho\left(f_{\lambda}\right)=$ $\sum_{v=0}^{\infty}\left(1 / p_{v}\right)\left|\lambda_{v}(T)\right|^{p_{v}}<\infty$, and $\left\|T-\lambda_{v}(T) I\right\|=0$ for all $v \in$ $\mathbb{N}$. We have $T=\lambda_{v}(T) I$, with $v \in \mathbb{N}$, hence $s_{v}(T)=s_{v}\left(\lambda_{v}(T\right.$ $I)=\left|\lambda_{v}(T)\right|$, with $v \in \mathbb{N}$. As a result, $f_{s} \in\left(H_{p(.)}\right)$, then $T$ $\in S_{\left(H_{p(.)}\right)_{p}}(\mathfrak{X}, \mathfrak{Y})$. Secondly, assume $\quad T \in S_{\left(H_{p(.)}\right)_{\rho}}(\mathfrak{X}, \mathfrak{Y})$. Hence, $f_{s} \in\left(H_{p(.)}\right)$, where $f_{s}(z)=\sum_{v=0}^{\infty} s_{v}(T) z^{v}$ converges for all $z \in \mathbb{C}$ with $\rho\left(f_{s}\right)=\sum_{v=0}^{\infty}\left(1 / p_{v}\right)\left|s_{v}(T)\right|^{p_{v}}<\infty$. One has

$$
\sum_{v=0}^{\infty} \frac{1}{p_{v}}\left|s_{v}(T)\right|^{p_{v}} \geq \frac{1}{\sup _{v} p_{v}} \sum_{v=0}^{\infty}\left[s_{v}(T)\right]^{p_{v}} .
$$

Therefore, $\lim _{v \longrightarrow \infty} s_{v}(T)=0$. Let $\left\|T-s_{v}(T) I\right\|^{-1}$ exists, for all $v \in \mathbb{N}$. Hence, $\left\|T-s_{v}(T) I\right\|^{-1}$ exists and bounded, for all $v \in \mathbb{N}$. Therefore, $\lim _{v \longrightarrow \infty}\left\|T-s_{v}(T) I\right\|^{-1}=\|T\|^{-1}$ exists and bounded. By using the prequasioperator ideal of $\left(S_{\left(\mathscr{L}_{p()}\right)_{p}}, g\right.$ ), one has

$$
I=T T^{-1} \in S_{\left(\mathscr{H}_{P(\cdot)}\right)_{\rho}}(\mathfrak{X}, \mathfrak{Y}) \Rightarrow\left(s_{v}(I)\right)_{v=0}^{\infty} \in\left(\mathscr{H}_{P(\cdot)}\right)_{\rho} \Rightarrow \lim _{\nu \longrightarrow \infty} s_{v}(I)=0 .
$$

Since $\lim _{v \longrightarrow \infty} s_{v}(I)=1$. Hence, $\left\|T-s_{v}(T) I\right\|=0$, for all $v$ $\in \mathbb{N}$. This gives $T \in\left(S_{\left(\mathscr{H}_{p(\cdot)}\right)_{\rho}}\right)^{\lambda}(\mathfrak{X}, \mathfrak{Y})$.

This shows the proof.

\section{Weighted Shift Operators on $\left(\mathscr{H}_{p(\cdot)}\right)_{\rho}$}

In this section, we present the upper bounds of $s$-numbers for infinite series of the weighted $n$th power forward and backward shift operator on $\mathscr{H}_{p(.)}$ with applications to some entire functions.

Theorem 36. Assume $\left(p_{v}\right) \in$ mi $\cap \cap \ell_{\infty}$ with $p_{0}>0$, then $V_{z}$ $\in L\left(\left(\mathscr{H}_{p(.)}\right)_{\rho}\right)$ with

$$
\left\|V_{z}\right\|=\sup _{r}\left(\frac{p_{r}}{p_{r+1}}\right)^{1 / \omega_{p}}
$$

where $\rho(f)=\left[\sum_{r=0}^{\infty}\left(1 / p_{r}\right)\left|f_{r} \wedge\right|^{p_{r}}\right]^{1 / \Phi_{p}}$, for all $f \in\left(\mathscr{H}_{p(.)}\right)$.

Proof. Suppose the setups are verified. For $f \in\left(H_{p(.)}\right)$. Since $\left(p_{v}\right) \in m i \nearrow \cap \ell_{\infty} \quad$ with $p_{0}>0$, then $\rho\left(V_{z} f\right)=\rho(z f)=$ $\left[\sum_{r=0}^{\infty}\left(1 / p_{r+1}\right)\left|f_{r} \wedge\right|^{p_{r+1}}\right]^{1 / \omega_{p}} \leq\left[\sum_{r=0}^{\infty}\left(1 / p_{r+1}\right)\left|f_{r} \wedge\right|^{p_{r+1}}\right]^{1 / \omega_{p}} \leq \sup _{r}$ $\left(p_{r} / p_{r+1}\right)^{1 / \omega_{p}}\left[\sum_{r=0}^{\infty}\left(1 / p_{r}\right)\left|f_{r} \wedge\right|^{p_{r+1}}\right]^{1 / \omega_{p}}=\sup \left(p_{r} / p_{r+1}\right)^{1 / \omega_{p}} \rho(f)$.

Therefore, $\quad V_{z} \in L\left(\left(\mathscr{H}_{p(\cdot)}\right)_{p}\right) \quad$ with $\quad\left\|V_{z}\right\| \leq \sup _{r}$ $\left(p_{r} / p_{r+1}\right)^{1 / \omega_{p}}$. Since $V_{z} \in L\left(\left(\mathscr{H}_{p(.)}\right)_{\rho}\right)$. Then, there is $A>0$ with $\rho\left(V_{z} f\right) \leq A \rho(f)$, for all $f \in\left(\mathscr{H}_{p(.)}\right)$. Hence, $\rho\left(V_{z} e^{(r)}\right)$ $\leq A \rho\left(e^{(r)}\right)$, one gets $\sup _{r}\left(p_{r} / p_{r+1}\right)^{1 / \omega_{p}} \leq\left\|V_{z}\right\|$.

This completes the proof.

Theorem 37. Consider $\left(p_{v}\right) \in$ mi $\cap \ell_{\infty}$ with $p_{0}>0$, then $B_{z}$ $\in L\left(\left(\mathscr{H}_{p(.)}\right)_{\rho}\right)$ with

$$
\left\|B_{z}\right\|=\sup _{r}\left(\frac{p_{r+1}}{p_{r}}\right)^{1 / \omega_{p}}
$$

where $\rho(f)=\left[\sum_{r=0}^{\infty}\left(1 / p_{r}\right)\left|f_{r} \wedge\right|^{p_{r}}\right]^{1 / \omega_{p}}$, for every $f \in\left(\mathscr{H}_{p(.)}\right)$.

Proof. Let the given settings hold for every $f \in\left(\mathscr{H}_{p(.)}\right)$. Since $\left(p_{v}\right) \in m i \nearrow \cap \ell_{\infty}$ with $p_{0}>0$, then

$$
\begin{aligned}
\rho\left(B_{z} f\right) & =\left[\sum_{r=0}^{\infty} \frac{1}{p_{r}}\left|f_{r+1} \wedge\right|^{p_{r}}\right]^{1 / \omega_{p}} \leq \sup _{r}\left(\frac{p_{r+1}}{p_{r}}\right)^{1 / \omega_{p}}\left[\sum_{r=0}^{\infty} \frac{1}{p_{r+1}}\left|f_{r+1} \wedge\right|^{p_{r}}\right]^{1 / \omega_{p}} \\
& \leq \sup _{r}\left(\frac{p_{r+1}}{p_{r}}\right)^{1 / \omega_{p}}\left[\sum_{r=0}^{\infty} \frac{1}{p_{r}}\left|f_{r} \wedge\right|^{p_{r}}\right]^{1 / \omega_{p}}=\sup _{r}\left(\frac{p_{r+1}}{p_{r}}\right)^{1 / \omega_{p}} \rho(f) .
\end{aligned}
$$


Therefore, $B_{z} \in L\left(\left(\mathscr{H}_{p(\cdot)}\right)_{\rho}\right)$ with $\left\|B_{z}\right\| \leq \sup _{r}\left(p_{r+1} / p_{r}\right)^{1 / \omega_{p}}$ . Since $B_{z} \in L\left(\left(\mathscr{H}_{p(.)}\right)\right)$. Then, there is $A>0$ with $\rho\left(B_{z} f\right) \leq$ $A \rho(f)$, for all $f \in\left(\mathscr{H}_{p(.)}\right)$. Hence, $\rho\left(B_{z} e^{(r+1)}\right) \leq A \rho\left(e^{(r+1)}\right)$, then $\sup _{r}\left(p_{r+1} / p_{r}\right)^{1 / \omega_{p}} \leq\left\|B_{z}\right\|$. This completes the proof.

Theorem 38. Let $\left(p_{v}\right) \in$ mi $\nearrow \cap \ell_{\infty}$ with $p_{0} \geq 1$. Suppose $\limsup _{v \longrightarrow \infty}\left(1 / \sqrt[v]{p_{v}}\right)=1$, then every function in $\left(\mathscr{H}_{p(.)}\right)_{\rho}$ is analytic on the open unit disc $\mathbb{D}$. Moreover, the convergence in $\left(\mathscr{H}_{p(\cdot)}\right)_{\rho}$ implies the uniform convergence on compact subsets of $\mathbb{D}$, where $\rho(f)=\left[\sum_{r=0}^{\infty}\left(1 / p_{r}\right)\left|f_{r} \wedge\right|^{p_{r}}\right]^{1 / \omega_{p}}$, for any $f \in$ $\left(\mathscr{H}_{p(\cdot)}\right)_{\rho} \cdot$

Proof. Suppose $\limsup _{v \longrightarrow \infty}\left(1 / \sqrt[v]{p_{v}}\right)=1$, and $f \in\left(\mathscr{H}_{p(\cdot)}\right)_{\rho}$. Therefore, $f(z)=\sum_{v=0}^{\infty} \widehat{v \longrightarrow \infty} \widehat{f}_{v}^{v}$ converges for every $z \in \mathbb{C}$ and $\rho(f)=$ $\left[\sum_{v=0}^{\infty}\left(1 / p_{v}\right)\left|f_{v} \wedge\right|^{p_{v}}\right]^{1 / \omega_{p}}<\infty . \quad$ Hence, $\quad \limsup _{v \longrightarrow \infty}$ $\sqrt[v]{\left(1 / p_{v}\right)\left|f_{v} \wedge\right|^{p_{v}}<1}$. We have

$$
\limsup _{v \longrightarrow \infty} \sqrt[v]{\left|f_{v} \wedge\right|^{p_{v}}}<\frac{1}{\lim \sup _{v \longrightarrow \infty}\left(1 / \sqrt[v]{p_{v}}\right)}=1 .
$$

Since $\left(p_{v}\right) \in m i \nearrow \cap \ell_{\infty}$ with $p_{0} \geq 1$, we obtain limsup $\sqrt[v]{\mid \widehat{f_{v}}}|| z|<| z|<| 1 \mid$, for all $z \in \mathbb{D}$. Hence, $f(z)=\sum_{v=0}^{\infty} \widehat{f_{v}} z^{v}$ converges for every complex value of $z \in D$. Assume $A$ is a compact subset of $\mathbb{D}$ and $f^{k}(z) \in A$, for all $k \in \mathbb{N}$. Let $f^{k}$ converges to $f \in\left(\mathscr{H}_{p(\bullet)}\right)_{\rho}$, we have

$$
\begin{aligned}
\left|f^{k}(z)-f(z)\right| & =\left|\sum_{v=0}^{\infty}\left(\widehat{f_{v}^{k}}-\widehat{f_{v}}\right) z^{v}\right| \leq \sum_{v=0}^{\infty}\left|\widehat{f_{v}^{k}}-\widehat{f_{v}}\right|\left|z^{v}\right| \\
& \leq\left[\sum_{v=0}^{\infty} \frac{1}{p_{v}}\left|f_{v}^{k} \wedge-f_{v} \wedge\right|^{p_{v}}\right]^{1 / \omega_{p}}\left[\sum_{v=0}^{\infty} p_{v}^{q_{v}}|z|^{v q_{v}}\right]^{1 / \omega_{p}} \\
& =\left[\sum_{v=0}^{\infty} p_{v}^{q_{v}}|z|^{v q_{v}}\right]^{1 / \omega_{p}} \rho\left(f^{k}-f\right)
\end{aligned}
$$

where $\left(q_{v}\right) \in$ mi $\nearrow \ell_{\infty}$ with $q_{0} \geq 1$ and $\left(1 / p_{v}\right)+\left(1 / q_{v}\right)=1$, for all $v \in \mathbb{N}$. Clearly, $\lim \sup p_{v}^{q_{v} / v}|z|^{q_{v}}<1$, then $\left[\sum_{v=0}^{\infty} p_{v}^{q_{v}}|z|^{v q_{v}}\right]^{1 / \omega_{p}}<\infty$. So $\lim _{k \longrightarrow \infty} f^{k}(z)=f(z) \in A$.

Theorem 39. Assume $V_{z}$ is the forward shift operator on $\left(\mathscr{H}_{p(\cdot)}\right)_{\rho}$, with $\rho(f)=\left[\sum_{r=0}^{\infty}\left(1 / p_{r}\right)\left|f_{r} \wedge\right|^{p_{r}}\right]^{1 / \omega_{p}}$, for all $f \in$
$\left(\mathscr{H}_{p(.)}\right)_{\rho}$. Then,

$\sup _{\operatorname{card} \xi=r+1} \inf _{k \in \xi}\left(\frac{p_{k}}{p_{k+n}}\right)^{1 / \omega_{p}} \frac{1}{A_{n}} \leq s_{r}\left(V_{z}^{n}\right) \leq \sup _{\operatorname{card}} \inf _{k=r+1}\left(\frac{p_{k}}{p_{k+n}}\right)^{1 / \omega_{p}}$,

where $A_{n}=\left[\sum_{k=0}^{\infty}\left(1 / p_{k}\right)\left|f_{k} \wedge\right|^{p_{k}}\right]^{1 / \omega_{p}} /\left[\sum_{k=\xi}^{\infty}\left(1 / p_{k}\right)\left|f_{k} \wedge\right|^{p_{k+n}}\right]^{1 / \omega_{p}}$.

Proof. Let card $\xi=r+1$ and as $V_{z}^{n} f \in\left(\mathscr{H}_{p(.)}\right)$, for all $f \in$ $\left(\mathscr{H}_{p(.)}\right)_{\rho}$, where $f(z)=\sum_{k=0}^{\infty} \widehat{f_{k}} z^{k}$ converges for every $z \in \mathbb{C}$ and $\rho(f)=\left[\sum_{k=0}^{\infty}\left(1 / p_{k}\right)\left|\overline{f_{k}}\right|^{p_{k}}\right]^{1 / \omega_{p}}<\infty$. Hence, $V_{z}^{n} f(z)=$ $\sum_{k=0}^{\infty} \widehat{f_{k}} z^{k+n}$ and $\rho\left(V_{z}^{n} f\right)=\left[\sum_{k=0}^{\infty}\left(a / p_{k+n}\right)\left|f_{k} \wedge\right|^{p_{k+n}}\right]^{1 / \omega_{p}}<\infty$ .Assume $P_{\xi}$ is and operator on $\left(\mathscr{H}_{p(\cdot)}\right)_{\rho}$ with rank $P_{\xi}=r+$ 1 defined by

$$
\left(p_{\xi} g\right)(z)=P_{\xi}\left(\sum_{k=0}^{\infty} \widehat{f_{k}} z^{k+n}\right)=\sum_{k \in \xi}^{i} \widehat{f_{k}} z^{k+n}
$$

Since $\quad \rho\left(P_{\xi} g\right)=\left[\sum_{k \in \xi}\left(1 / p_{k+n}\right)\left|f_{k} \wedge\right|^{p_{k+n}}\right]^{1 / \omega_{p}} \leq$ $\left[\sum_{k=0}^{\infty}\left(1 / p_{k+n}\right)\left|f_{k} \wedge\right|^{p_{k+n}}\right]^{1 / \omega_{p}}=\rho(g)$. This implies $\left\|P_{\xi}\right\| \leq 1$.

Define an operator $S_{z}^{n}$ by $\left(S_{z}^{n} h\right)(z)=S_{z}^{n}\left(\sum_{k \in \xi} \widehat{f_{k}} z^{k+n}\right)=$ $\sum_{k=0}^{\infty} \widehat{f_{k}} z^{k}$, then

$$
\rho\left(S_{z}^{n} h\right)=\left[\sum_{k=0}^{\infty} \frac{1}{p_{k}}\left|f_{k} \wedge\right|^{p_{k}}\right]^{1 / \omega_{p}} \leq U_{n}\left[\sum_{k \in \xi} \frac{1}{p_{k+n}}\left|f_{k} \wedge\right|^{p_{k+n}}\right]^{1 / \omega_{p}}=U_{n} \rho(h) .
$$

Hence, $\left\|S_{z}^{n}\right\| \leq U_{n}$, where

$$
1 \leq U_{n}=\frac{\left[\sum_{k=0}^{\infty}\left(1 / p_{k}\right)\left|f_{k} \wedge\right|^{p_{k}}\right]^{1 / \omega_{p}}}{\left[\left.\sum_{k \in \xi}\left|1 / p_{k+n}\right| f_{k} \wedge\right|^{p_{k+n}}\right]^{1 / \Theta_{p}}}<\infty
$$

Therefore, the identity map will be $I_{r+1}=P_{\xi} V_{z}^{n} S_{z}^{n}$, according to the definition of $s$-numbers, we have

$$
\begin{gathered}
s_{r}\left(I_{r+1}\right)=1 \leq\left\|P_{\xi}\right\| s_{r}\left(V_{z}^{n}\right)\left\|s_{z}^{n}\right\| \leq s_{r}\left(V_{z}^{n}\right)\left\|s_{z}^{n}\right\| \Rightarrow \\
s_{r}\left(V_{z}^{n}\right) \geq \frac{1}{\left\|S_{z}^{n}\right\|} \leq \frac{1}{U_{n}}=\frac{\left[\left.\sum_{k \in \xi}\left|1 / p_{k+n}\right| f_{k} \wedge\right|^{p_{k+n}}\right]^{1 / \omega_{p}}}{\left[\sum_{k=0}^{\infty}\left(1 / p_{k}\right)\left|f_{k} \wedge\right|^{p_{k}}\right]^{1 / \omega_{p}}} \geq \inf _{k \in \xi}\left(\frac{p_{k}}{p_{k+n}}\right)^{1 / \omega_{p}} \frac{1}{A_{n}} .
\end{gathered}
$$
has

This inequality is satisfied for all card, $\xi=r+1$ and one

$$
s_{r}\left(V_{z}^{n}\right) \geq \sup _{\operatorname{card}} \inf _{\xi=r+1}\left(\frac{p_{k}}{p_{k+n}}\right)^{1 / \oplus_{p}} \frac{1}{A_{n}} .
$$

On the other hand, let $\xi$ be a subset of $\mathbb{N}$ with card $\xi=r$. Define the finite rank map $R_{z}^{n}$ by $\left(R_{z}{ }^{n} v\right)(z)=R_{z}^{n}\left(\sum_{k=0}^{\infty} \widehat{f_{k}} z^{k}\right)$ $=\sum_{k \in \xi} \widehat{f}_{k} z^{k+n}$. In view of the definition of approximation 
numbers, we have

$$
\begin{aligned}
s_{r}\left(V_{z}^{n}\right) & \leq \alpha_{r}\left(V_{z}^{n}\right) \leq\left\|V_{z}^{n}-R_{z}^{n}\right\| \leq \sup _{|f(z)| \neq 0} \frac{\left|\left(V_{z}^{n}-R_{z}^{n}\right) f(z)\right|}{|f(z)|} \\
& =\sup _{|f(z)| \neq 0} \frac{\left|\sum_{k \notin \xi} \widehat{f}_{k} z^{k+n}\right|}{|f(z)|} \leq \sup _{|f(z)| \neq 0} \frac{\left[\sum_{k \notin \xi}\left(1 / p_{k+n}\right)\left|\widehat{f}_{k}\right|^{p_{k+n}}\right]^{1 / \omega_{p}}}{|f(z)|} \\
& \leq \sup _{k \notin \xi}\left(\frac{p_{k}}{p_{k+n}}\right)^{1 / \omega_{p}} .
\end{aligned}
$$

This inequality is verified for every card $\xi=r$ and by using Lemma 10, one has

$$
\begin{aligned}
& \sup _{\text {card } \xi=r+1} \inf _{k \in \xi}\left(\frac{p_{k}}{p_{k+n}}\right)^{1 / \omega_{p}} \frac{1}{A_{n}} \leq s_{r}\left(V_{z}^{n}\right) \leq \inf _{\operatorname{card}} \xi \sup _{k \notin \xi}\left(\frac{p_{k}}{p_{k+n}}\right)^{1 / \omega_{p}} \\
& =\sup _{\operatorname{card}} \inf _{\xi=r+1}\left(\frac{p_{k}}{p_{k+n}}\right)^{1 / \omega_{p}} .
\end{aligned}
$$

This completes the proof.

Theorem 40. If $B_{z}$ is the backward shift operator on $\left(\mathscr{H}_{p(.)}\right)$, with $\rho(f)=\left[\sum_{r=0}^{\infty}\left(1 / p_{r}\right)\left|\widehat{f}_{r}\right|^{p_{r}}\right]^{1 / \omega_{p}}$, for all $f \in\left(\mathscr{H}_{p(.)}\right)_{\rho}$ Then, $\sup _{\operatorname{card} \xi=r+1} \inf _{k \in \xi}\left(\frac{p_{k+n}}{p_{k}}\right)^{1 / \omega_{p}} \frac{1}{G_{n}} \leq s_{r}\left(B_{z}^{n}\right) \leq \sup _{\operatorname{card} \xi=r+1} \inf _{k \in \xi}\left(\frac{p_{k+n}}{p_{k}}\right)^{1 / \omega_{p}}$

where $G_{n}=\left[\sum_{k=0}^{\infty}\left(1 / p_{k}\right)\left|f_{k} \wedge\right|^{p_{k}}\right]^{1 / \omega_{p}} /\left[\left.\sum_{k \in \xi}\left|1 / p_{k+n}\right| f_{k} \wedge\right|^{p_{k+n}}\right]^{1 / \omega_{p}}$.

Proof. Assume card $\xi=r+1$ and since $B_{z}^{n} f \in\left(\mathscr{H}_{p(.)}\right)_{\rho}$, for every $f \in\left(\mathscr{H} p_{(.)}\right)$, where $f(z)=\sum_{k=0}^{\infty} \widehat{f}_{k} z^{k}$ converges for any $z \in \mathbb{C}$ and $\rho(f)=\left[\sum_{k=0}^{\infty}\left(1 / p_{k}\right)\left|f_{k} \wedge\right|^{p_{k}}\right]^{1 / \omega_{p}}<\infty$. Therefore, $B_{z}^{n} f(z) \sum_{k=0}^{\infty} \widehat{f}_{k+n} z^{k}$ and $\rho\left(B_{z}^{n} f\right)=\left[\sum_{k=0}^{\infty}\left(1 / p_{k}\right)\left|f_{k} \wedge\right|^{p_{k}}\right]^{1 / \omega_{p}}<\infty$ .Suppose $P_{\xi}$ is an operator on $\left(\mathscr{H}_{p(.)}\right)_{\rho}$ with rank $P_{\xi}=r+1$ evident by

$$
\left(p_{\xi} g\right)(z)=P_{\xi}\left(\sum_{k=0}^{\infty} \widehat{f}_{k+n} z^{k}\right)=\sum_{k \in \xi}^{\infty} \widehat{f}_{k+n} z^{k}
$$

As

$$
\rho(P \xi g)=\left[\sum_{k \in \xi}\left(1 / p_{k}\right)\left|\widehat{f}_{k+n}\right|^{p_{k}}\right]^{1 / \omega_{p}} \leq
$$

$\left[\sum_{k=0}^{\infty}\left(1 / p_{k}\right)\left|f_{k+n} \wedge\right|^{p_{k}}\right]^{1 / \omega_{p}}=p(g)$. This implies $\left\|p_{\xi}\right\| \leq 1$.

Define an operator $S_{z}^{n}$ by $\left(S_{z}^{n} h\right)(z)=S_{z}^{n}\left(\sum_{k \in \xi} \widehat{f}_{k+n} z^{k}\right)=\sum_{k=0}^{\infty}$ $\widehat{f}_{k} z^{k}$, one gets

$$
\rho\left(S_{z}^{n} h\right)=\left[\sum_{k=0}^{\infty} \frac{1}{p_{k}}\left|\widehat{f}_{k}\right|^{p_{k}}\right]^{1 / \omega_{p}} \leq U_{n}\left[\sum_{k \in \xi}\left|\widehat{f}_{k+n}\right|^{p_{k}}\right]^{1 / \omega_{p}}=U_{n} \rho(h) .
$$

Therefore, $\quad\left\|S_{z}^{n}\right\| \leq U_{n}, \quad$ where $\quad 1 \leq U_{n}=$ $\left[\sum_{k=0}^{\infty}\left(1 / p_{k}\right)\left|\widehat{f}_{k}\right|^{p_{k}}\right]^{1 / \omega_{p}} /\left[\left.\sum_{k \in \xi}\left|\left(1 / p_{k}\right)\right| \widehat{f}_{k+n}\right|^{p_{k}}\right]^{1 / \omega_{p}}<\infty$. Hence, the identity operator will be $I_{r+1}=P_{\xi} B_{z}^{n} S_{z}^{n}$, in view of the definition of $s$-numbers, one has

$$
\begin{gathered}
s_{r}\left(I_{r+1}\right)=1 \leq\left\|P_{\xi}\right\| s_{r}\left(B_{z}^{n}\right)\left\|S_{z}^{n}\right\| \leq s_{r}\left(B_{z}^{n}\right)\left\|S_{z}^{n}\right\| \Rightarrow \\
s_{r}\left(B_{z}^{n}\right) \geq \frac{1}{\left\|S_{z}^{n}\right\|} \geq \frac{1}{U_{n}}=\frac{\left[\left.\sum_{k \in \xi}\left|\left(1 / p_{k}\right)\right| \widehat{f}_{k+n}\right|^{p_{k}}\right]^{1 / \omega_{p}}}{\left[\sum_{k=0}^{\infty}\left(1 / p_{k}\right)\left|\widehat{f}_{k}\right|^{p_{k}}\right]^{1 / \omega_{p}}} \geq \inf _{k \in \xi}\left(\frac{p_{k+n}}{p_{k}}\right)^{1 / \omega_{p}} \frac{1}{G_{n}} .
\end{gathered}
$$
have

This inequality is confirmed for all card $\xi=r+1$, and we

$$
s_{r}\left(B_{z}^{n}\right) \geq \sup _{\operatorname{card}} \inf _{\xi=r+1}\left(\frac{p_{k+n}}{p_{k}}\right)^{1 / \omega_{p}} \frac{1}{G_{n}} .
$$

On the other hand, suppose $\xi$ is a subset of $\mathbb{N}$ with card $\xi=r$. Define the finite rank operator $R_{z}^{n}$ by $\left(R_{z}^{n} v\right)(z)=R_{z}^{n}($ $\left.\sum_{k=0}^{\infty} \widehat{f}_{k} z^{k}\right)=\sum_{k \in \xi} \widehat{f}_{k+n} z^{k}$. From the definition of approximation numbers, one gets

$$
\begin{aligned}
s_{r}\left(B_{z}^{n}\right) & \leq \alpha_{r}\left(B_{z}^{n}\right) \leq \| B_{z}^{n}-R_{z}^{n}|| \leq \sup _{|f(z)| \neq 0} \frac{\left|\left(B_{z}^{n}-R_{z}^{n}\right) f(z)\right|}{|f(z)|} \\
& =\sup _{|f(z)| \neq 0} \frac{\left|\sum_{k \notin \xi} \widehat{f}_{k+n} z^{k}\right|}{|f(z)|} \leq \sup _{|f(z)| \neq 0} \frac{\left[\sum_{k \notin \xi} 1 / p_{k}\left|\widehat{f}_{k+n}\right|^{p_{k}}\right]^{1 / \omega_{p}}}{|f(z)|} \\
& \leq \sup _{k \notin \xi}\left(\frac{p_{k+n}}{p_{k}}\right)^{1 / \omega_{p}} .
\end{aligned}
$$

This inequality is satisfied for any card $\xi=r$ and from Lemma 10, we have

$$
\begin{aligned}
& \sup _{\operatorname{card} \xi=r+1} \inf _{k \in \xi}\left(\frac{p_{k+n}}{p_{k}}\right)^{1 / \omega_{p}} \frac{1}{G_{n}} \leq s_{r}\left(B_{z}^{n}\right) \leq \inf _{\operatorname{card} \xi=r} \sup _{k \in \xi}\left(\frac{p_{k+n}}{p_{k}}\right)^{1 / \omega_{p}} \\
& \quad=\sup _{\operatorname{card} \xi=r+1} \inf _{k \in \xi}\left(\frac{p_{k+n}}{p_{k}}\right)^{1 / \omega_{p}} .
\end{aligned}
$$

This finishes the proof. 
Next, the upper and lower bounds of norm $\sum_{m=0}^{\infty} c_{m} V_{z}^{m}$ on the space $\left(\mathscr{H} p_{(.)}\right)_{\rho}$ have been explained.

Theorem 41. The effect of $\sum_{m=0}^{\infty} c_{m} V_{z}^{m}$ on the space $\left(\mathscr{H}_{p(.)}\right)_{p}$, where $\rho(f)=\left[\sum_{r=0}^{\infty} 1 / p_{r}\left|\widehat{f}_{r}\right|^{p_{r}}\right]^{1 / \omega_{p}}$, for all $f \in\left(\mathscr{H}_{p(.)}\right)$, $\left(c_{m}\right)_{m=0}^{\infty} \in \ell^{(p m) / \omega_{p}}$, and $\left(p_{v}\right) \in m i \nearrow \cap \ell_{\infty}$ with $p_{0} \geq 1$, we have

$$
\begin{gathered}
\sup _{k}\left[\sum_{m=0}^{\infty}\left|c_{m}\right|^{p_{m+k}} \frac{p_{k}}{p_{k+m}}\right]^{1 / \omega_{p}} \leq\left\|\sum_{m 0}^{\infty} c_{m} V_{z}^{m}\right\| \\
\quad \leq \sup _{m, k}\left(\frac{p_{k}}{p_{k+m}}\right)^{1 / \omega_{p}} \sum_{m=0}^{\infty}\left|c_{m}\right|^{p_{m} / \omega_{p}} .
\end{gathered}
$$

Proof. Assume $f \in\left(\mathscr{H}_{p(.)}\right)$, we have $\sum_{m=0}^{\infty} c_{m} V_{z}^{m} f(z)=\sum_{k=0}^{\infty}$ $\sum_{m=0}^{\infty} c_{m} \widehat{f}_{k} z^{k+m}$. Then,

$$
\begin{aligned}
\left\|\sum_{m=0}^{\infty} c_{m} V_{z}^{m}\right\| & \geq \frac{\rho\left(\sum_{m=0}^{\infty} c_{m} V_{z}^{m} e^{(k)}\right)}{\rho\left(e^{(k)}\right)}=\left[\frac{\sum_{m=0}^{\infty}\left(1 / p_{m+k}\right)\left|c_{m}\right|^{p_{m+k}}}{1 / p_{k}}\right]^{1 / \omega_{p}} \\
& \geq \sup _{k}\left[\sum_{m=0}^{\infty}\left|c_{m}\right|^{p_{m+k}} \frac{p_{k}}{p_{k+m}}\right]^{1 / \omega_{p}} .
\end{aligned}
$$

Since $\rho$ satisfies the triangle inequality, we get

$$
\begin{aligned}
\left\|\sum_{m=0}^{\infty} c_{m} V_{z}^{m}\right\| & =\sup _{p(f) \neq 0} \frac{\rho\left(\sum_{m=0}^{\infty} c_{m} V_{z}^{m} f\right)}{\rho(f)} \\
& \leq \sup _{p(f) \neq 0} \frac{\sum_{m=0}^{\infty}\left[\sum_{k=0}^{\infty}\left(1 / p_{m+k}\right)\left(\left|c_{m}\right|\left|\widehat{f}_{k}\right|\right)^{p_{m+k}}\right]^{1 / \omega_{p}}}{\left[\sum_{k=0}^{\infty}\left(1 / p_{k}\right)\left|\widehat{f}_{k}\right|^{p_{k}}\right]^{1 / \omega_{p}}} \\
& \leq \sup _{m, k}\left(\frac{p_{k}}{p_{k+m}}\right)^{1 / \omega_{p}} \frac{\sum_{m=0}^{\infty}\left[\sum_{k=0}^{\infty}\left(1 / p_{k}\right)\left(\left|c_{m}\right|\left|\widehat{f}_{k}\right|\right)^{p_{m+k}}\right]^{1 / \omega_{p}}}{\left[\sum_{k=0}^{\infty}\left(1 / p_{k}\right)\left(1 / p_{k}\right)\left|\widehat{f}_{k}\right|^{p_{k}}\right]^{1 / \omega_{p}}} \\
& \leq \sup _{m, k}\left(\frac{p_{k}}{p_{k+m}}\right)^{1 / \omega_{p}} \sum_{m=0}^{\infty}\left|c_{m}\right|^{p_{m} / \omega_{p}} .
\end{aligned}
$$

Next the upper and lower bounds of norm $\sum_{m=0}^{\infty} c_{m} B_{z}^{m}$ on the space $\left(\mathscr{H}_{p(.)}\right)_{\rho}$ have been investigated.

Theorem 42. The effect of $\sum_{m=0}^{\infty} c_{m} B_{z}^{m}$ on the space $\left(\mathscr{H}_{p(.)}\right)$, where $\rho(f)=\left[\sum_{r=0}^{\infty} 1 / p_{r}\left|\widehat{f}_{r}\right|^{p_{r}}\right]^{1 / \omega_{p}}$, for all $f \in\left(\mathscr{H}_{p(.)}\right),\left(c_{m}\right)_{m=0}^{\infty}$ $\in \ell^{\left(p_{m}\right) / \omega_{p}}$, and $\left(p_{v}\right) \in$ mi $\nearrow \cap \ell_{\infty}$ with $p_{0} \geq 1$, we have

$\sup _{k}\left[\sum_{m=0}^{\infty}\left|c_{m}\right|^{p_{k}} \frac{p_{k+m}}{p_{k}}\right]^{1 / \omega_{p}} \leq\left\|\sum_{m=0}^{\infty} c_{m} B_{z}^{m}\right\| \leq \sup _{m, k}\left(\frac{p_{k+m}}{p_{k}}\right)^{1 / \omega_{p}} \sum_{m=0}^{\infty}\left|c_{m}\right|^{p_{m} / \omega_{p}}$.

Proof. Suppose $f \in\left(\mathscr{H}_{p(.)}\right)$, one has $\sum_{m=0}^{\infty} c_{m} B_{z}^{m} f(z)=\sum_{k=0}^{\infty}$ $\sum_{m=0}^{\infty} c_{m} \widehat{f}_{k+m} z^{k}$. We have

$$
\begin{aligned}
\left\|\sum_{m=0}^{\infty} c_{m} B_{z}^{m}\right\| & \geq \frac{\rho\left(\sum_{m=0}^{\infty} c_{m} B_{z}^{m} e^{(k)}\right)}{\rho\left(e^{(k)}\right)}=\left[\frac{\sum_{m=0}^{\infty}\left(1 / p_{k-m}\right)\left|c_{m}\right|^{p_{k-m}}}{1 / p_{k}}\right]^{1 / \omega_{p}} \\
& \geq \sup _{k}\left[\sum_{m=0}^{\infty}\left|c_{m}\right|^{p_{k}} \frac{p_{k+m}}{p_{k}}\right]^{1 / \omega_{p}} .
\end{aligned}
$$

As $\rho$ verifies the triangle inequality, one can see

$$
\begin{aligned}
\left\|\sum_{m=0}^{\infty} c_{m} B_{z}^{m}\right\| & =\sup _{p(f) \neq 0} \frac{\rho\left(\sum_{m=0}^{\infty} c_{m} B_{z}^{m} f\right)}{\rho(f)} \\
& \leq \sup _{p(f) \neq 0} \frac{\sum_{m=0}^{\infty}\left[\sum_{k=0}^{\infty}\left(1 / p_{k}\right)\left(\left|c_{m}\right|\left|\widehat{f}_{k+m}\right|\right)^{p_{k}}\right]^{1 / \omega_{p}}}{\left[\sum_{k=0}^{\infty}\left(1 / p_{k}\right)\left|\widehat{f}_{k}\right|^{p_{k}}\right]^{1 / \omega_{p}}} \\
& \leq \sup _{m, k}\left(\frac{p_{k+m}}{p_{k}}\right)^{1 / \omega_{p}} \frac{\sum_{m=0}^{\infty}\left[\sum_{k=0}^{\infty}\left(1 / p_{k+m}\right)\left(\left|c_{m}\right|\left|\widehat{f}_{k+m}\right|\right)^{p_{k}}\right]^{1 / \omega_{p}}}{\left[\sum_{k=0}^{\infty}\left(1 / p_{k}\right)\left|\widehat{f}_{k}\right|^{p_{k}}\right]^{1 / \omega_{p}}} \\
& \leq \sup _{m, k}\left(\frac{p_{k+m}}{p_{k}}\right)^{1 / \omega_{p}} \sum_{m=0}^{\infty}\left|c_{m}\right|^{p_{m} / \omega_{p} .}
\end{aligned}
$$

The following theorem indicates an upper estimation to the $s$-numbers of $\sum_{m=0}^{\infty} c_{m} V_{z}^{m}$ on the space $\left(\mathscr{H} p_{(.)}\right)$.

Theorem 43. The effect of $\sum_{m=0}^{\infty} c_{m} V_{z}^{m}$ on the space $\left(\mathscr{H} p_{(.)}\right)$, where $p(f)=\left[\sum_{r=0}^{\infty} 1 / p_{r}\left|\widehat{f}_{r}\right|^{p_{r}}\right]^{1 / \omega_{p}}$, for all $f \in\left(\mathscr{H} p_{(.)}\right)$, the $s$ -numbers of this operator are presented by

$$
s_{r}\left(\sum_{m=0}^{\infty} c_{m} V_{z}^{m}\right) \leq \sup _{\operatorname{card} \xi=r+1} \inf _{k \in \xi} \sup _{m}\left(\frac{p_{k}}{p_{k+m}}\right)^{1 / \omega_{p}} \sum_{m=0}^{\infty}\left|c_{m}\right|^{p_{m} / \omega_{p}},
$$

for all $\left(c_{m}\right)_{m=0}^{\infty} \in \ell^{\left(p_{m}\right) / \omega_{p}}$ and $\left(p_{v}\right) \in m i, \cap \ell_{\infty}$ with $p_{0} \geq 1$.

Proof. Let $\xi$ be a subset of $\mathbb{N}$ and card $\xi=r$. By using the definition of $s$-numbers. Define the finite rank operator $R$ by $R$ $f(z)=R\left(\sum_{k=0}^{\infty} \widehat{f}_{k} z^{k}\right)=\sum_{k \in \xi} \sum_{m=0}^{k} c_{m} \widehat{f}_{k-m} z^{k}$. In view of the definition of approximation numbers and since $\rho$ satisfies 
the triangle inequality, we have

$$
\begin{aligned}
s_{r}\left(\sum_{m=0}^{\infty} c_{m} V_{z}^{m}\right) & \leq \alpha_{r}\left(\sum_{m=0}^{\infty} c_{m} V_{z}^{m}\right) \leq\left\|\sum_{m=0}^{\infty} c_{m} V_{z}^{m}-R\right\| \\
& \leq \sup _{\rho(f) \neq 0} \frac{\rho\left(\sum_{m=0}^{\infty} c_{m} V_{z}^{m} f-R f\right)}{\rho(f)} \\
& \leq \sup _{\rho(f) \neq 0} \frac{\sum_{m=0}^{\infty}\left[\sum_{k \notin \xi}\left(1 / p_{m+k}\right)\left(\left|c_{m}\right|\left|\hat{f}_{k}\right|\right)^{p_{k+m}}\right]^{1 / \omega_{p}}}{\rho(f)} \\
& \leq \sup _{k \notin \xi, m}\left(\frac{p_{k}}{p_{k+m}}\right)^{1 / \omega_{p}} \sum_{m=0}^{\infty}\left|c_{m}\right|^{p_{m} / \omega_{p}} .
\end{aligned}
$$
has

This inequality is verified for every card $\xi=r$, and one

$$
\begin{aligned}
s_{r}\left(\sum_{m=0}^{\infty} c_{m} V_{z}^{m}\right) & \leq \inf _{\text {card } \xi=r} \sup _{k \notin \xi, m}\left(\frac{p_{k}}{p_{k+m}}\right)^{1 / \omega_{p}} \sum_{m=0}^{\infty}\left|c_{m}\right|^{p_{m} / \omega_{p}} \\
& =\sup _{\operatorname{card} \xi=r+1} \inf _{k \notin \xi} \sup _{m}\left(\frac{p_{k}}{p_{k+m}}\right)^{1 / \omega_{p}} \sum_{m=0}^{\infty}\left|c_{m}\right|^{p_{m} / \omega_{p}} .
\end{aligned}
$$

This implies the proof.

The next theorem investigates an upper estimation to the $s$-numbers of $\sum_{m=0}^{\infty} c_{m} B_{z}^{m}$ on the space $\left(\mathscr{H}_{p(.)}\right)_{\rho}$.

Theorem 44. Acting $\sum_{m=0}^{\infty} c_{m} B_{z}^{m}$ on the space $\left(\mathscr{H}_{p_{(.)}}\right)_{\rho}$, where $\rho(f)=\left[\sum_{r=0}^{\infty}\left(1 / p_{r}\right)\left|\widehat{f}_{r}\right|^{p_{r}}\right]^{1 / \varrho_{p}}$, for every $f \in\left(\mathscr{H}_{p(.)}\right)_{\rho}$, the $s$ -numbers of this operator satisfy

$$
s_{r}\left(\sum_{m=0}^{\infty} c_{m} B_{z}^{m}\right) \leq \sup _{\operatorname{card}} \inf _{k=r+1} \sup _{m}\left(\frac{p_{k+m}}{p_{k}}\right)^{1 / \omega_{p}} \sum_{m=0}^{\infty}\left|c_{m}\right|^{p_{m} / \omega_{p}}
$$

for all $\left(c_{m}\right)_{m=0}^{\infty} \in \ell^{\left(p_{m}\right) / \omega_{p}}$ and $\left(p_{v}\right) \in$ mi $\cap \cap \ell_{\infty}$ with $p_{0} \geq 1$.

Proof. Assume $\xi$ is a subset of $\mathbb{N}$ and card $\xi=r$. From the definition of $s$-numbers. Define the finite rank operator $R b$ $y R f(z)=R\left(\sum_{k=0}^{\infty} \widehat{f}_{k} z^{k}\right)=\sum_{k \in \xi} \sum_{m=0}^{k} c_{m} \widehat{f}_{k-m} z^{k}$. From the definition of approximation numbers and as $\rho$ verifies the trian- gle inequality, one has

$$
\begin{aligned}
s_{r}\left(\sum_{m=0}^{\infty} c_{m} B_{z}^{m}\right) & \leq \alpha_{r}\left(\sum_{m=0}^{\infty} c_{m} B_{z}^{m}\right) \leq\left\|\sum_{m=0}^{\infty} c_{m} B_{z}^{m}-R\right\| \\
& \leq \sup _{\rho(f) \neq 0} \frac{\rho\left(\sum_{m=0}^{\infty} c_{m} B_{z}^{m} f-R f\right)}{\rho(f)} \\
& \leq \sup _{\rho(f) \neq 0} \frac{\sum_{m=0}^{\infty}\left[\sum_{k \notin \xi}\left(1 / p_{k}\right)\left(\left|c_{m}\right|\left|\widehat{f}_{k+m}\right|\right)^{p_{k}}\right]^{1 / \omega_{p}}}{\rho(f)} \\
& \leq \sup _{k \notin \xi, m}\left(\frac{p_{k+m}}{p_{k}}\right)^{1 / \omega_{p}} \sum_{m=0}^{\infty}\left|c_{m}\right|^{p_{m} / \omega_{p}} .
\end{aligned}
$$

This inequality is satisfied for all card $\xi=r$, and we have

$$
\begin{aligned}
s_{r}\left(\sum_{m=0}^{\infty} c_{m} B_{z}^{m}\right) & \leq \inf _{\text {card } \xi=r} \sup _{k \notin \xi, m}\left(\frac{p_{k+m}}{p_{k}}\right)^{1 / \omega_{p}} \sum_{m=0}^{\infty}\left|c_{m}\right|^{p_{m} / \omega_{p}} \\
& =\sup _{\text {card } \xi=r+1} \inf _{k \notin \xi} \sup _{m}\left(\frac{p_{k+m}}{p_{k}}\right)^{1 / \omega_{p}} \sum_{m=0}^{\infty}\left|c_{m}\right|^{p_{m} / \omega_{p}} .
\end{aligned}
$$

This completes the proof.

The following theorems are direct consequences of Theorem 43 and Definition 18, for some entire functions, for example, the exponential and the sine functions.

Theorem 45. Let $\left(p_{v}\right) \in m i, \cap \ell_{\infty}$ with $p_{0} \geq 1$. Assume $B_{e^{z}}$ is a shift operator on $\left(\mathscr{H}_{p_{(.)}}\right)_{\rho}$, where for $\rho(f)=$ $\left[\sum_{r=0}^{\infty}\left(1 / p_{r}\right)\left|\widehat{f}_{r}\right|^{p_{r}}\right]^{1 / \Phi_{p}}$, for all $f \in\left(\mathscr{H}_{p(.)}\right)_{\rho}$ and $e^{z}=\sum_{m=0}^{\infty} z^{m} / m !$ . The upper estimation of the s-number of $V_{e^{z}}$ is given by

$$
s_{r}\left(V_{e^{z}}\right) \leq \sup _{\text {card } \xi=r+1} \inf _{k \in \xi} \sup _{m}\left(\frac{p_{k+m}}{p_{k}}\right)^{1 / \omega_{p}} \sum_{m=0}^{\infty}\left(\frac{1}{m !}\right)^{p_{m} / \Phi_{p}} .
$$

Theorem 46. Let $\left(p_{v}\right) \in m i, \cap \ell_{\infty}$ with $p_{0} \geq 1$. Suppose $B_{\sin }(z)$ is a shift operator on $\left(\mathscr{H}_{p_{(.)}}\right)_{\rho}$, where $\rho(f)=$ $\left[\sum_{r=0}^{\infty}\left(1 / p_{r}\right)\left|\widehat{f}_{r}\right|^{p_{r}}\right]^{1 / \Phi_{p}}$, for all $f \in\left(\mathscr{H}_{p(.)}\right)_{\rho}$ and $\sin (z)=\sum_{m=0}^{\infty}$ $(-1)^{m}\left(z^{2 m+1} /(2 m+1) !\right)$. The upper estimation of the $s$ numbers of $V_{\sin (z)}$ is presented by

$$
s_{r}\left(V_{\sin (z)}\right) \leq \sup _{c a r d \xi=r+1} \inf _{k \in \xi} \sup _{m}\left(\frac{p_{k+m}}{p_{k}}\right)^{1 / \omega_{p}} \sum_{m=0}^{\infty}\left(\frac{1}{(2 m+1) !}\right)^{p_{m} / \omega_{p}} .
$$

The following theorems are direct consequences of Theorem 44 and Definition 19, for some entire functions, for example, the exponential and the sine functions. 
Theorem 47. Assume $\left(p_{v}\right) \in m i, \cap \ell_{\infty}$ with $p_{0} \geq 1$. Suppose $B_{e^{z}}$ is a shift operator on $\left(\mathscr{H}_{p_{(.)}}\right)$, where $\rho(f)=$ $\left[\sum_{r=0}^{\infty}\left(1 / p_{r}\right)\left|\widehat{f}_{r}\right|^{p_{r}}\right]^{1 / \omega_{p}}$, for every $f \in\left(\mathscr{H}_{p(.)}\right)$ and $e^{z}=\sum_{m=0}^{\infty} z^{m} /$ $m !$. The upper estimation of the s-numbers of $B_{e^{z}}$ is pretended by

$$
s_{r}\left(B_{e^{z}}\right) \leq \sup _{\text {card } \xi=r+1} \inf _{k \in \xi} \sup _{m}\left(\frac{p_{k+m}}{p_{k}}\right)^{1 / \omega_{p}} \sum_{m=0}^{\infty}\left(\frac{1}{m !}\right)^{p_{m} / \omega_{p}} .
$$

Theorem 48. Suppose $\left(p_{v}\right) \in m i, \cap \ell_{\infty}$ with $p_{0} \geq 1$. Assume $B_{\sin (z)}$ is a shift operator on $\left(\mathscr{H}_{p_{(.)}}\right)_{\rho}$, where $\rho(f)=$ $\left[\sum_{r=0}^{\infty}\left(1 / p_{r}\right)\left|\widehat{f}_{r}\right|^{p_{r}}\right]^{1 / \omega_{p}}$, for every $f \in\left(\mathscr{H}_{p(.)}\right)_{\rho}$ and $\sin (z)=$ $\sum_{m=0}^{\infty}(-1)^{m}\left(z^{2 m+1} /(2 m+1) !\right)$. The upper estimation of the $B_{\sin (z)}$ is presented by

$s_{r}\left(B_{\sin (z)}\right) \leq \sup _{\operatorname{card} \xi=r+1} \inf _{k \in \xi} \sup _{m}\left(\frac{p_{k+m}}{p_{k}}\right)^{1 / \Phi_{p}} \sum_{m=0}^{\infty}\left(\frac{1}{(2 m+1) !}\right)^{p_{m} / \Phi_{p}}$.

\section{Data Availability}

No data were used.

\section{Conflicts of Interest}

The authors declare that they have no competing interests.

\section{Authors' Contributions}

All authors contributed equally to the writing of this paper. All authors read and approved the final manuscript.

\section{Acknowledgments}

This work was funded by the University of Jeddah, Saudi Arabia, under grant No. UJ-20-080-DR. The authors, therefore, acknowledge with thanks the University's technical and financial support.

\section{References}

[1] A. A. Bakery and M. H. El Dewaik, "A generalization of Caristi's fixed point theorem in the variable exponent weighted formal power series space," Journal of Function Spaces, vol. 2021, Article ID 9919420, 18 pages, 2021.

[2] A. Pietsch, Operator Ideals, North-Holland Publishing Company, Amsterdam-New York-Oxford, 1980.

[3] A. Pietsch, "Small ideals of operators," Studia Mathematica, vol. 51, no. 3, pp. 265-267, 1974.

[4] B. M. Makarov and N. Faried, "Some properties of operator ideals constructed by $s$ numbers (In Russian)," in Theory of Operators in Functional Spaces, pp. 206-211, Academy of Science, Siberian section, Novosibirsk, Russia, 1977.
[5] N. Faried and A. A. Bakery, "Small operator ideals formed by $s$ numbers on generalized Cesáro and Orlicz sequence spaces," Journal of Inequalities and Applications, vol. 2018, no. 1, 2018.

[6] A. L. Shields, "Weighted shift operators and analytic function theory," in Topics of Operator Theory, in: Mathematical Surveys Monographs, vol. 13, American Mathematical Society, Providence, RI, USA, 1974.

[7] K. Hedayatian, "On cyclicity in the space $H^{P}(\beta)$," Taiwanese Journal of Mathematics, vol. 8, no. 3, pp. 429-442, 2004.

[8] H. Emamirad and G. S. Heshmati, "Chaotic weighted shifts in Bargmann spaces," Journal of mathematical analysis and applications, vol. 308, no. 1, pp. 36-46, 2005.

[9] N. Faried, A. Morsy, and Z. A. Hassanain, "s-numbers of shift operators of formal entire functions," Journal of Approximation Theory, vol. 176, pp. 15-22, 2013.

[10] B. E. Rhoades, "Operators of $A-p$ type," Atti della Accademia Nazionale dei Lincei. Classe di Scienze Fisiche, Matematiche e Naturali. Rendiconti, vol. 59, no. 3-4, pp. 238-241, 1975.

[11] A. Pietsch, Eigenvalues and S-Numbers, Cambridge University Press, New York, NY, USA, 1986.

[12] A. A. Bakery and A. R. Abou Elmatty, "A note on Nakano generalized difference sequence space," Advances in Difference Equations, vol. 2020, no. 1, 17 pages, 2020.

[13] N. Faried, Z. Abd El Kader, and A. A. Mehanna, "s-numbers of polynomials of shift operators on $\ell^{p} 1 \leq p \leq \infty$," Journal of the Egyptian Mathematical Society, vol. 1, pp. 31-37, 1993.

[14] B. Altay and F. Başar, "Generalization of the sequence space $\ell(p)$ derived by weighted means," Journal of Mathematical Analysis and Applications, vol. 330, no. 1, pp. 147-185, 2007.

[15] A. Pietsch, Operator Ideals, VEB Deutscher Verlag derWissenschaften, Berlin, 1978.

[16] A. Pietsch, "s-numbers of operators in Banach spaces," Studia Mathematica, vol. 51, no. 3, pp. 201-223, 1974. 